\title{
STOCHASTIC TWO DIMENSIONAL EULER EQUATIONS
}

\author{
By ZdZISŁAW BRZÉ́NIAK AND SZYMON PESZAT ${ }^{1}$ \\ University of Hull and Polish Academy of Sciences
}

\begin{abstract}
The existence of a martingale solution to 2-dimensional stochastic Euler equations is proved. The constructed solution is a limit as the viscosity converges to zero of a sequence of solutions to modified Navier-Stokes equations.
\end{abstract}

1. Introduction. Let $\mathscr{O}$ be a smooth open subset of $\mathbb{R}^{2}$. The paper is concerned with the existence of a martingale solution to the stochastic incompressible Euler equations

$$
\left\{\begin{array}{l}
\partial_{t} u+\langle u, \nabla\rangle u+\nabla p=F(t, u)+G(t, u) \dot{W} \\
\operatorname{div} u=0
\end{array}\right.
$$

with the boundary condition

$$
\langle u, \mathbf{n}\rangle=0 \quad \text { on } \partial \mathscr{O} \text {, }
$$

where $\mathbf{n}$ stands for the unit outward normal to $\partial \mathscr{O}$. Further we assume that the initial value $u_{0}$ satisfies $\operatorname{div} u_{0}=0$ on $\mathscr{O}$ and $\left\langle u_{0}, \mathbf{n}\right\rangle=0$ on $\partial \mathscr{O}$.

In (1.1), $W$ is a cylindrical Wiener process on a real separable Hilbert space $\mathscr{H}$, and $u=\left(u_{1}, u_{2}\right)$ and $p$ are unknown random fields. We interpret $u(t, x)=$ $\left(u_{1}(t, x), u_{2}(t, x)\right)$ and $p(t, x)$ as the velocity and pressure of an incompressible perfect fluid, and $F(t, v)+G(t, u) \dot{W}$ as the density of the external random force per unit volume.

We will formulate our main existence result in a rather abstract form. However it covers the following two cases. The equations on $\mathscr{O}=\mathbb{R}^{2}$ driven by an $\mathbb{R}^{m}$-valued spatially homogeneous random field $W=\left(W_{1}, \ldots, W_{m}\right)$; see Section 2 . In this case $\partial \mathscr{O}=\emptyset$, and so the boundary condition $\langle u, \mathbf{n}\rangle=0$ can be dropped. The second important case is the equations with two independent $L^{2}\left(\mathscr{O} ; \mathbb{R}^{m}\right)$-valued Wiener processes. In both cases the mappings $F$ and $G$ can be of the Nemytski form; see Theorems 2.2 and 2.3.

Received October 1999; revised March 2001.

${ }^{1}$ Supported in part by KBN Grant 2PO3A 06408 and by an EPSRC Visiting Fellowship Research Grant at the University of Hull.

AMS 2000 subject classifications. Primary 35Q05, 35Q30, 30H15, 60G60; secondary 35R60, 60J25.

Key words and phrases. Stochastic Euler equations, stochastic Navier-Stokes equations, stochastic hydromechanics, martingale problems on Banach spaces. 
Let $\nu>0$ and $T>0$. We will show that the following linearized modified Navier-Stokes equations on $[0, T] \times \mathscr{O}$,

$$
\left\{\begin{array}{l}
\partial_{t} u^{(\nu, n)}-\nu \Delta u^{(\nu, n)}+\left\langle u^{(\nu, n)}, \nabla\right\rangle \varphi_{n}\left(u^{(\nu, n)}\right)+\nabla p^{(\nu, n)} \\
\quad=F_{n}\left(t, u^{(\nu, n)}\right)+G_{n}\left(t, u^{(\nu, n)}\right) \dot{W} \\
\operatorname{div} u^{(\nu, n)}=0 \\
u^{(\nu, n)}(0)=u_{0}^{(n)}
\end{array}\right.
$$

with the boundary conditions

$$
\left\langle u^{(\nu, n)}, \mathbf{n}\right\rangle=0 \quad \text { and } \quad \operatorname{curl} u^{(\nu, n)}=0 \quad \text { on } \partial \mathscr{O}
$$

has a unique solution. In (1.3), $F_{n}, G_{n}$ and $u_{0}^{(n)}$ are some regularizations of $F$, $G$ and $u_{0}$, and $\varphi_{n}$ is a bounded and Lipschitz function in appropriate functional spaces; see Section 5 for details. We will construct the solution to (1.1)-(1.2) proving first the weak compactness of the family $\left\{u^{(\nu, n)}, \nu>0, n \in \mathbb{N}\right\}$ in $L^{2}\left(0, T ; L^{q}\left(\mathscr{O}, e^{-|x|} \mathrm{d} x ; \mathbb{R}^{2}\right)\right)$. We will use some ideas of Gątarek-Gołdys [24], see also [12], [16], [17]. The crucial point of the proof is to show that for any $p \in[2, \infty)$ one has the following estimate in the Sobolev spaces $W^{1,2}:=$ $W^{1,2}\left(\mathscr{O} ; \mathbb{R}^{2}\right)$ and $W^{1, q}:=W^{1, q}\left(\mathscr{O} ; \mathbb{R}^{2}\right)$,

$$
\sup _{\nu>0} \sup _{n} \mathbb{E} \sup _{t \in[0, T]}\left(\left\|u^{(\nu, n)}(t)\right\|_{W^{1,2}}^{p}+\left\|u^{(\nu, n)}(t)\right\|_{W^{1, q}}^{p}\right)<\infty .
$$

To do this we adopt the method developed for the deterministic Euler equations, see [3], [29], [30] and [33]. In this method an important role is played by the operator $\operatorname{curl} v(x):=\partial_{2} v_{1}(x)-\partial_{1} v_{2}(x)$.

Stochastic Euler equations with periodic boundary conditions were considered in [15] using nonstandard analysis. The problem on a bounded domain was considered in [6], [7] and [8]. In the present paper we treat equations on a possibly unbounded region, and using the theory of stochastic integration in Banach spaces we obtain better regularity of solutions. In particular, for suitable initial values we can show their space Hölder continuity; see Remark 2.2.

The paper is organized as follows. In Section 2 we introduce the notation and we formulate our main results on the existence of a solution to (1.1) - (1.2). In Section 3 we formulate two theorems on analytical properties of the modified Stokes operator on $L^{q}$-spaces, and the nonlinear term $\langle u, \nabla\rangle u$ appearing in the Euler and Navier-Stokes equations. These theorems are proven in Appendix A. In Section 4 we present basic facts concerning stochastic integration in Banach, in particular $W^{r, q}$ spaces. We also evaluate the so-called $\gamma$-radonifying norm of a $W^{r, q}$-valued integral operator, see Theorem 4.1. The proof of this result is given in Appendix B. Section 5 is devoted to the prove of the estimate (1.5) for solutions to the modified stochastic Navier-Stokes equations (1.3) - (1.4). In the next section we prove our main result; Theorem 2.1. In the following two sections we derive from Theorem 2.1 the existence of solutions to the stochastic Euler equations driven by a spatially homogeneous Wiener random field and by an $L^{2}\left(\mathscr{O} ; \mathbb{R}^{m}\right)$-valued Wiener process. 
2. Notation and formulation of results. Let us denote by $C_{0}^{\infty}\left(\overline{\mathscr{O}} ; \mathbb{R}^{d}\right)$, where $d=1,2$, the space consisting of all mappings $v \in C\left(\overline{\mathscr{O}} ; \mathbb{R}^{d}\right) \cap C^{\infty}\left(\mathscr{O} ; \mathbb{R}^{d}\right)$ such that $\operatorname{supp} v:=\overline{\{x \in \overline{\mathscr{O}}: v(x) \neq 0\}}$ is compact in $\overline{\mathscr{O}}$. Let $C_{\text {sol }}^{\infty}$ be the collections of all $v \in C_{0}^{\infty}\left(\overline{\mathscr{O}} ; \mathbb{R}^{2}\right)$ satisfying the boundary and incompressibility conditions: $\langle v, \mathbf{n}\rangle=0$ on $\partial \mathscr{O}$ and $\operatorname{div} v=0$ in $\mathscr{O}$. For $q \in(1, \infty)$ let $\mathbb{X}_{q}$ be the closure of $C_{\text {sol }}^{\infty}$ in $L^{q}:=L^{q}\left(\mathscr{O} ; \mathbb{R}^{2}\right)$, and let $W^{1, q}$ be the closure of $C_{0}^{\infty}\left(\overline{\mathscr{O}} ; \mathbb{R}^{2}\right)$ with respect to the norm

$$
\left(\int_{\mathscr{O}}\left(|v(x)|^{q}+|\nabla v(x)|^{q}\right) \mathrm{d} x\right)^{1 / q} .
$$

Denote the space $\mathbb{X}_{q} \cap W^{1, q}$ by $H^{1, q}$. Note that $H^{1, q}$ is a closed subspace of $W^{1, q}$ and that the original norm on $H^{1, q}$ is equivalent to the one induced by $W^{1, q}$.

Assume that $W$ is a cylindrical Wiener process on a real separable Hilbert space $\mathscr{H}$. Let us denote by $R\left(\mathscr{H}, W^{1, q}\right)$ the space of all $\gamma$-radonifying mappings acting from $\mathscr{H}$ into $W^{1, q}$. For the convenience of the reader we will recall in Section 4 the notion of a cylindrical Wiener processes, radonifying operators and the radonifying norm. Furthermore, we present basic facts on the theory of stochastic integration in Banach spaces. In the definition below, and also in the whole paper, $\langle\cdot, \cdot\rangle$ stands for the scalar product in $L^{2}$, or $\mathbb{R}^{2}$. We denote by $L_{(\mathrm{HS})}\left(\mathscr{H}, W^{1,2}\right)$ the space of all Hilbert-Schmidt operators acting from $\mathscr{H}$ into $W^{1,2}$. In what follows we fix $T>0$.

DeFinition 2.1. Let $u_{0} \in H^{1,2} \cap H^{1, q}$ for $q \in[2, \infty)$. Let $F$ and $G$ be jointly measurable mappings acting from $[0, T] \times\left(H^{1,2} \cap H^{1, q}\right)$ into $W^{1,2} \cap W^{1, q}$ and $L_{(\mathrm{HS})}\left(\mathscr{H}, W^{1,2}\right) \cap R\left(\mathscr{H}, W^{1, q}\right)$, respectively.

A martingale $H^{1,2} \cap H^{1, q}$-valued solution to the stochastic Euler problem (1.1) - (1.2) is a triple consisting of a filtered probability space $\mathscr{I}=(\Omega, \mathscr{F}$, $\left.\left(\mathscr{F}_{t}\right)_{t \in[0, T]}, \mathbb{P}\right)$, an $\left(\mathscr{F}_{t}\right)$-adapted cylindrical Wiener process $W(t), t \geq 0$, on $\mathscr{H}$ and an $\left(\mathscr{F}_{t}\right)$-adapted measurable $H^{1,2} \cap H^{1, q}$-valued process $u(t), t \geq 0$, such that:

(i) for every $p \in[1, \infty), u \in L^{p}\left(\Omega ; L^{\infty}\left(0, T ; H^{1,2} \cap H^{1, q}\right)\right)$;

(ii) for all $z \in C_{\text {sol }}^{\infty}$ and $t \in[0, T]$ one has a.s.

$$
\begin{aligned}
\langle u(t), z\rangle= & \left\langle u_{0}, z\right\rangle+\int_{0}^{t}\left\{\sum_{i=1}^{2}\left\langle u_{i}(s) u(s), \nabla z_{i}\right\rangle+\langle F(s, u(s)), z\rangle\right\} \mathrm{d} s \\
& +\left\langle\int_{0}^{t} G(s, u(s)) \mathrm{d} W(s), z\right\rangle .
\end{aligned}
$$

REMARK 2.1. In the definition of the solution the incompressibility and boundary conditions are contained in the requirement that $u$ belong to $H^{1,2} \cap$ $H^{1, q}$. Since $\operatorname{div} u=0$, the term $\left\langle u, \nabla u_{i}\right\rangle$ has been replaced by $\operatorname{div}\left(u u_{i}\right)$. Note that the gradient of the pressure vanishes after projecting the both sides of (1.1) onto the divergence free space, and that (ii) is the weak, in the sense of 
PDEs, form of the projected equation. For if we take the inner product in $L^{2}$ of both sides of (0.1) with $z$ such that $\operatorname{div} z=0$, then the pressure term vanishes and after integrating by parts we obtain (ii). Going back, suppose that $u$ is sufficiently regular. We move all the terms of (ii) on one side and integrate by parts. Then we arrive at the relation of the form

$$
\left\langle u(t)-u(0)-\int_{0}^{t} \Theta(s ; u) \mathrm{d} s, z\right\rangle=0 \quad \forall z: \operatorname{div} z=0,
$$

with a certain $\Theta$. Since we have the following orthogonal decomposition

$$
L^{2}=\overline{\left\{\nabla p: p \in C_{0}^{\infty}\left(\mathscr{O} ; \mathbb{R}^{2}\right)\right\}} \oplus \overline{\left\{\psi \in C_{0}^{\infty}\left(\mathscr{O} ; \mathbb{R}^{2}\right): \operatorname{div} \psi=0\right\}},
$$

we conclude that $u(t)-u(0)-\int_{0}^{t} \Theta(s ; u) \mathrm{d} s$ is in the form of the gradient of a certain function, say $\Phi(t)$. Hence formally $\nabla p(t)=\partial_{t} \Phi(t)$, however $\Phi$ need not to be absolutely continuous with respect to the time variable, and consequently we allow the case of $\nabla p$ being a distribution in $t$. One can show that $\Phi$ is absolutely continuous with respect to $t$ under the additional assumption that $G(t, u) \psi, \psi \in \mathscr{H}$ is divergence free.

Let us denote by $H_{L^{2}}^{1,2}$ the space $H^{1,2}$ endowed with the strong $L^{2}$-topology. The $L^{2}$-norm is denoted by $|\cdot|$. Recall that $C_{\mathrm{sol}}^{\infty}$ stands for the space of smooth divergence free mappings satisfying boundary condition (1.2). We impose the following standing assumption on the set $\mathscr{O}$.

ASSUMPTION A. Either $\mathscr{O}=\mathbb{R}^{2}$ or the Dirichlet problem in $\mathscr{O}$ is well posed in the following sense. For any $f \in L^{q}(\mathscr{O})$ there exists a unique $v \in W_{0}^{1, q}(\mathscr{O}) \cap$ $W^{2, q}(\mathscr{O})$ such that

$$
-\Delta v=f \text { in } D \quad \text { and } \quad v=0 \text { on } \partial D .
$$

Denote by $\hat{B}_{q}$ a linear operator in $L^{q}(\mathscr{O})$ defined by: $D\left(\hat{B}_{q}\right)=W_{0}^{1, q}(\mathscr{O}) \cap$ $W^{2, q}(\mathscr{O}), \hat{B}_{q} v=-\Delta v$. Then we assume that $\hat{B}_{q}$ has bounded imaginary powers, that is, there exist constants $\varepsilon<\frac{\pi}{2}$ and $C_{\varepsilon}<\infty$ such that $\left\|\hat{B}_{q}^{i s}\right\|_{L\left(L^{q}(\mathscr{O}), L^{q}(\mathscr{O})\right)} \leq$ $C_{\varepsilon} \mathrm{e}^{\varepsilon|s|}, s \in \mathbb{R}$. Finally, we assume that if $f \in W^{1, q}(\mathscr{O})$, then the solution $u$ to the problem above belongs to $W^{3, q}(\mathscr{O})$

Let us observe that in view of well celebrated results of Seeley [42], the second part of the assumption A is satisfied when $\mathscr{O}$ is a bounded domain with $\mathscr{C}^{\infty}$ boundary. The case with $b^{2}$ boundary is studied by Prüss and Sohr in [40]. The third part of the assumption is satisfied if in addition the boundary $\partial \mathscr{O}$ is of $\mathscr{b}^{3}$ class, see [2] and [46].

The main result of the present paper is:

THEOREM 2.1. Let $q \in[2, \infty)$. Assume that:

(i) $F:[0, T] \times H^{1,2} \rightarrow W^{1,2}, F:[0, T] \times H^{1, q} \rightarrow W^{1, q}$, and $G:[0, T] \times$ $H^{1,2} \rightarrow L_{(\mathrm{HS})}\left(\mathscr{H}, W^{1,2}\right), G:[0, T] \times H^{1, q} \rightarrow R\left(\mathscr{H}, W^{1, q}\right)$ are jointly measurable, and there are functions $a_{1} \in L^{1}(0, T)$ and $a_{2} \in L^{r}(0, T)$ with $r>2$, such 
that for all $t \in[0, T]$ and $v \in H^{1,2} \cap H^{1, q}$ one has

$$
\begin{aligned}
&\|F(t, v)\|_{W^{1,2}} \leq a_{1}(t)\left(1+\|v\|_{H^{1,2}}\right), \\
&\|F(t, v)\|_{W^{1, q}} \leq a_{1}(t)\left(1+\|v\|_{H^{1, q}}\right), \\
&\|G(t, v)\|_{L_{(\mathrm{HS})}\left(\mathscr{C}, W^{1,2}\right)} \leq a_{2}(t)\left(1+\|v\|_{H^{1,2}}\right), \\
&\|G(t, v)\|_{R\left(\mathscr{C}, W^{1, q}\right)} \leq a_{2}(t)\left(1+\|v\|_{H^{1, q}}\right) ;
\end{aligned}
$$

(ii) for all $t \in[0, T]$, and $z \in C_{\text {sol }}^{\infty}$ the real valued functions $v \mapsto\langle F(t, v), z\rangle$ and $v \mapsto\left|G^{*}(t, v) z\right|_{\mathscr{H}}$ are continuous on $H_{L^{2}}^{1,2}$. Then for any $u_{0} \in H^{1,2} \cap H^{1, q}$ there exists a martingale $H^{1,2} \cap H^{1, q}$-valued solution to the problem (1.1)-(1.2).

REMARK 2.2. If $q>2$ then by the Sobolev imbedding theorem $W^{1, q}$ is continuously imbedded into the space of Hölder continuous mappings $C^{\alpha}, \alpha<$ $1-2 / q$. Thus, as $H^{1, q}$ is a subspace of $W^{1, q}$ we can get the space continuity of the solution to (1.1)-(1.2).

The remaining part of this section is concerned with the application of Theorem 2.1 to the case of $F$ and $G$ being Nemytski operators and $W$ being either an $\mathbb{R}^{m}$-valued spatially homogeneous Wiener random field, or an $L^{2}\left(\mathscr{O} ; \mathbb{R}^{m}\right)$ valued Wiener process with a nuclear covariance operator. We will need the following two definitions.

Definition 2.2. Let $q \in[2, \infty)$, and $r \in[1, \infty)$. A mapping $h:[0, T] \times \mathscr{O} \times$ $\mathbb{R}^{2} \rightarrow \mathbb{R}^{2}$ belongs to the class $U(\mathscr{O}, q, r)$ iff $h(t, x, y)=h^{(1)}(t, x)+h^{(2)}(t, x, y)$, $t \in[0, T], x \in \mathscr{O}, y \in \mathbb{R}^{2}$, where:

(i) $h^{(1)}$ and $h^{(2)}$ are measurable, and for any $t \in[0, T], h^{(1)}(t, \cdot) \in W^{1,2} \cap W^{1, q}$ and $h^{(2)}(t, \cdot, \cdot)$ is differentiable;

(ii) there are functions $a \in L^{r}(0, T)$ and $\phi \in L^{2}(\mathscr{O}) \cap L^{q}(\mathscr{O})$, such that all $t \in[0, T]$, and $x \in \mathscr{O}, y \in \mathbb{R}^{2}$,

$$
\begin{aligned}
\left|h^{(1)}(t, \cdot)\right|_{W^{1,2}}+\left|h^{(1)}(t, \cdot)\right|_{W^{1, q}} & \leq a(t), \\
\left|h^{(2)}(t, x, y)\right|+\sum_{i=1}^{2}\left|\partial_{x_{i}} h^{(2)}(t, x, y)\right| & \leq a(t)(\phi(x)+|y|), \\
\sum_{i=1}^{2}\left|\partial_{y_{i}} h^{(2)}(t, x, y)\right| & \leq a(t) .
\end{aligned}
$$

We say that $h:[0, T] \times \mathscr{O} \times \mathbb{R}^{2} \rightarrow \mathbb{R}^{2}$ belongs to the class $U(\mathscr{O}, \infty, r)$ iff it is differentiable with respect to the second and third variables, and there is a function $a \in L^{r}(0, T)$ such that

$$
\mid h\left(t, x, y \mid+\sum_{i=1}^{2}\left\{\left|\partial_{x_{i}} h(t, x, y)\right|+\left|\partial_{y_{i}} h(t, x, y)\right|\right\} \leq a(t), \quad t \in[0, T], x \in \mathscr{O}, y \in \mathbb{R}^{2} .\right.
$$


DEFINITION 2.3. Let $\mathscr{\Re}$ be a filtered probability space. By an $\mathbb{R}^{m}$-valued spatially homogeneous Wiener random field on $[0, T] \times \mathbb{R}^{2}$ we understand a system $\mathscr{W}_{j}, j=1, \ldots, m$ of independent measurable real valued random fields on $[0, T] \times \mathbb{R}^{2}$ such that each $\mathscr{W}_{j}$ is Gaussian, Wiener and spatially homogeneous, that is:

(i) the random vector $\left(\mathscr{W}_{j}\left(t_{1}, x_{1}\right), \ldots, \mathscr{W}_{j}\left(t_{n}, x_{n}\right)\right)$ is Gaussian for an arbitrary finite sequence $\left(t_{1}, x_{1}\right), \ldots,\left(t_{n}, x_{n}\right) \in[0, T] \times \mathbb{R}^{2}$;

(ii) for each $x \in \mathbb{R}^{2},\left\{\mathscr{W}_{j}(t, x)\right\}_{t \in[0, T]}$ is a real valued Wiener process with respect to the filtration $\left(\mathscr{F}_{t}\right)$;

(iii) for arbitrary $t \in[0, T], n \in \mathbb{N}, x_{1}, \ldots, x_{n} \in \mathbb{R}^{2}$ and $h \in \mathbb{R}^{2}$ the random vectors $\left(\mathscr{W}_{j}\left(t, x_{1}+h\right), \ldots, \mathscr{W}_{j}\left(t, x_{n}+h\right)\right)$ and $\left(\mathscr{W}_{j}\left(t, x_{1}\right), \ldots, \mathscr{W}_{j}\left(t, x_{n}\right)\right)$ have the same distribution.

Let $\mathscr{W}$ be an $\mathbb{R}^{m}$-valued spatially homogeneous Wiener random field on $\mathbb{R}^{2}$. Then (see [38]) there are symmetric positive finite measures $\mu_{j}, j=1, \ldots, m$ on $\mathbb{R}^{2}$ such that for all $j=1, \ldots, m, t, s \in[0, T]$, and $x, y \in \mathbb{R}^{2}$ one has

$$
\mathbb{E} \mathscr{W}_{j}(t, x) \mathscr{W}_{j}(s, y)=t \wedge s \frac{1}{2 \pi} \int_{\mathbb{R}^{2}} \exp \{i\langle x-y, z\rangle\} \mathrm{d} \mu_{j}(z) .
$$

We call $\mu=\left(\mu_{1}, \ldots, \mu_{m}\right)$ the spectral measure of $\mathscr{W}$. Clearly, the law of $\mathscr{W}$ is uniquely determined by its spectral measure.

Let $\mathscr{W}$ be an $\mathbb{R}^{m}$-valued spatially homogeneous Wiener random field on $\mathbb{R}^{2}$ with a spectral measure $\mu$, and let $f, g^{j}:[0, T] \times \mathbb{R}^{2} \times \mathbb{R}^{2} \rightarrow \mathbb{R}^{2}, j=1, \ldots, m$. Consider the following system of equations on $[0, T] \times \mathbb{R}^{2}$,

$$
\left\{\begin{array}{l}
\partial_{t} u+\langle u, \nabla\rangle u+\nabla p=f(t, x, u)+\sum_{j=1}^{m} g^{j}(t, x, u) \dot{\mathscr{W}}_{j}(t, x), \\
\operatorname{div} u=0 .
\end{array}\right.
$$

By a martingale $H^{1,2} \cap H^{1, q}$-valued solution to (2.1) we understand a triple consisting of a filtered probability space $\Re$, an $\left(\mathscr{F}_{t}\right)$-adapted $\mathbb{R}^{m}$-valued spatially homogeneous Wiener process $\mathscr{W}$ with the spectral measure $\mu$, and an $\left(\mathscr{F}_{t}\right.$ )-adapted $H^{1,2} \cap H^{1, q}$-valued process $u$ satisfying conditions (i) to (ii) from Definition 2.1. In the integral equation in (ii) we replace $F(t, u)$ by $f(t, \cdot, u(\cdot))$ and we write

$$
\left\langle\int_{0}^{t} G(s, u(s)) \mathrm{d} W(s), z\right\rangle=\sum_{j=1}^{m} \int_{\mathbb{R}^{2}}\left\langle\int_{0}^{t} g^{j}(s, x, u(s, x)) \mathscr{W}_{j}(\mathrm{~d} s, x), z(x)\right\rangle \mathrm{d} x,
$$

where $\mathscr{W}_{j}(\mathrm{~d} t, x)$ means that for fixed $x$ we integrate in Itô's sense with respect to the real-valued Wiener process $\mathscr{W}_{j}(\cdot, x)$, see [21].

THEOREM 2.2. Let $q \in[2, \infty)$. Assume that:

(i) $\sum_{j=1}^{m} \int_{\mathbb{R}^{2}}\left(1+|y|^{q}\right) \mathrm{d} \mu_{j}(y)<\infty$;

(ii) $f$ belongs to $U\left(\mathbb{R}^{2}, q, 1\right)$ and $g^{j}, j=1, \ldots, m$ belong to $U\left(\mathbb{R}^{2}, q, r\right)$ for a certain $r>2$. Then for any $u_{0} \in H^{1,2} \cap H^{1, q}$ there exists a martingale $H^{1,2} \cap H^{1, q}$-valued solution to the problem (2.1) such that $u(0)=u_{0}$ a.s. 
REMARK 2.3. The random field $\mathscr{W}$ can be viewed as a cylindrical Wiener process on a properly chosen Hilbert space $\mathscr{H}_{\mathscr{W}}$, see Example 4.1 in Section 4 , or [38]. We note that $\mathscr{H}_{\mathscr{W}}$ is a function space. In fact, it is a subspace of the space on which $\mathscr{W}$ lives. Thus in particular, $\mathscr{H}_{\mathscr{W}} \subset L^{2}\left(\mathbb{R}^{2}, \mathrm{e}^{-|x|} \mathrm{d} x ; \mathbb{R}^{m}\right)$. Equation (2.1) can be written as a stochastic evolution equation with $F$ and $G$ given by

$$
F(t, u)(x)=f(t, x, u(x)), \quad(G(t, u) \psi)(x)=\sum_{j=1}^{m} g^{j}(t, x, u(x)) \psi_{j}(x),
$$

where $\psi=\left(\psi_{1}, \ldots, \psi_{m}\right) \in \mathscr{H}_{\mathscr{W}}$. Thus we are in the framework of Definition 2.1. In the last section we show that $F$ and $G$ satisfy the assumptions (i) and (ii) of Theorem 2.1 with $\mathscr{H}=\mathscr{H}_{\mathscr{W}}$, and consequently that Theorem 2.2 is a special case of Theorem 2.1.

DEFINITION 2.4. An $L^{2}\left(\mathscr{O} ; \mathbb{R}^{m}\right)$-valued process $W$ defined on a filtered probability space $\mathscr{R}$ is called Wiener iff for any $\psi \in L^{2}\left(\mathscr{O} ; \mathbb{R}^{m}\right),\langle W(t), \psi\rangle_{L^{2}\left(\mathscr{O} ; \mathbb{R}^{m}\right)}$, $t \in[0, T]$, is a real-valued Wiener process.

Let $W$ be an $L^{2}\left(\mathscr{O} ; \mathbb{R}^{m}\right)$-valued Wiener process. Then (see, e.g., [19], [33]), there is a symmetric non-negative trace class operator $Q$ on $L^{2}\left(\mathscr{O} ; \mathbb{R}^{m}\right)$ such that for all $u, v \in L^{2}\left(\mathscr{O} ; \mathbb{R}^{m}\right)$ and $t, s \in[0, T]$ one has

$$
\mathbb{E}\langle W(t), u\rangle_{L^{2}\left(\mathscr{O} ; \mathbb{R}^{m}\right)}\langle W(s), v\rangle_{L^{2}\left(\mathscr{O} ; \mathbb{R}^{m}\right)}=t \wedge s\langle Q u, v\rangle_{L^{2}\left(\mathscr{O} ; \mathbb{R}^{m}\right)} .
$$

We call $Q$ the covariance operator of $W$. Since $Q^{1 / 2}$ is Hilbert-Schmidt it is given by an integral kernel $\mathscr{Q}$, that is

$$
Q^{1 / 2} \psi(x)=\left(\sum_{l=1}^{m} \int_{\mathscr{O}} \mathscr{Q}_{1, l}(x, y) \psi_{l}(y) \mathrm{d} y, \ldots, \sum_{l=1}^{m} \int_{\mathscr{O}} \mathscr{Q}_{m, l}(x, y) \psi_{l}(y) \mathrm{d} y\right)
$$

Let $\mathscr{O}$ be a smooth connected open subset of $\mathbb{R}^{2}$ satisfying Assumption A, and let $W, \tilde{W}$ be two independent $L^{2}\left(\mathscr{O} ; \mathbb{R}^{m}\right)$-valued Wiener processes with the covariance operators $Q$ and $\tilde{Q}$, respectively. We denote by $\mathscr{Q}$ and $\tilde{\mathscr{Q}}$ the integral kernels corresponding to $Q^{1 / 2}$ and $\tilde{Q}^{1 / 2}$. Let $f, g^{j}, \tilde{g}^{j}:[0, T] \times \mathscr{O} \times \mathbb{R}^{2} \rightarrow \mathbb{R}^{2}$. Consider the stochastic Euler equations on $[0, T] \times \mathscr{O}$,

$$
\begin{aligned}
\partial_{t} u+\langle u, \nabla\rangle u+\nabla p= & f(t, x, u) \\
& +\sum_{j=1}^{m}\left\{g^{j}(t, x, u) \dot{W}_{j},(t)+\tilde{g}^{j}(t, x, u) \dot{\tilde{W}}_{j}(t)\right\}, \\
\operatorname{div} u= & 0,
\end{aligned}
$$

with the boundary condition

$$
\langle u, \mathbf{n}\rangle=0 \quad \text { on } \partial \mathscr{O} .
$$

The processes $W$ and $\tilde{W}$ can be treated as cylindrical on some Hilbert spaces $\mathscr{H}_{W}$ and $\mathscr{H}_{\tilde{W}}$, see Section 4. By a solution to (2.4)-(2.5) we understand a solution 
to the problem (1.1) - (1.2) with $\mathscr{H}=\mathscr{H}_{W} \times \mathscr{H}_{\tilde{W}}, F$ given by (2.2), and $G$ defined for $x \in \mathscr{O}$, and $\psi=(\varphi, \tilde{\varphi}) \in \mathscr{H}$ by the formula

$$
(G(t, u) \psi)(x)=\sum_{j=1}^{m}\left\{g^{j}(t, x, u(x)) \varphi_{j}(x)+\tilde{g}^{j}(t, x, u(x)) \tilde{\varphi}_{j}(x)\right\}
$$

THEOREM 2.3. Let $q \in[2, \infty)$. Assume that:

(i) the integral kernels $\mathscr{Q}$ and $\tilde{\mathscr{Q}}$ satisfy the estimates

$$
\begin{aligned}
\sum_{l, j=1}^{m} \sum_{|\alpha| \leq 1} \sup _{x \in \mathscr{O}} \int_{\mathscr{O}}\left(\partial_{x}^{\alpha} \mathscr{Q}_{i, j}(x, y)\right)^{2} \mathrm{~d} y<\infty, & \sum_{l, j=1}^{m} \sum_{|\alpha| \leq 1}\left[\int_{\mathscr{O}} \int_{\mathscr{O}}\left(\partial_{x}^{\alpha} \tilde{\mathscr{Q}}_{i, j}(x, y)\right)^{2} \mathrm{~d} y \mathrm{~d} x\right. \\
& \left.+\int_{\mathscr{O}}\left(\int_{\mathscr{O}}\left(\partial_{x}^{\alpha} \tilde{\mathscr{Q}}_{i, j}(x, y)\right)^{2} \mathrm{~d} y\right)^{q / 2} \mathrm{~d} x\right]<\infty,
\end{aligned}
$$

(ii) $f$ belongs to $U(\mathscr{O}, q, 1), g^{j}, j=1, \ldots, m$ belong to $U(\mathscr{O}, q, r)$ for a certain $r>2$, and $\tilde{g}^{j}, j=1, \ldots, m$ belong to $U(\mathscr{O}, \infty, r)$ for a certain $r>2$. Then for any $u_{0} \in H^{1,2} \cap H^{1, q}$ there exists a martingale $H^{1,2} \cap H^{1, q}$-valued solution to $(2.4)-(2.5)$ such that $u(0)=u_{0}$ a.s.

3. Analytical preliminaries. From now on, we use the notation $L^{q}$ indiscriminately for the spaces $L^{q}(\mathscr{O})$ or $L^{q}\left(\mathscr{O} ; \mathbb{R}^{2}\right)$. Similarly, $W^{r, q}, q \in(1, \infty)$, $r \in \mathbb{R}$ stand for the Sobolev spaces $W^{r, q}(\mathscr{O})$ or $W^{r, q}\left(\mathscr{O} ; \mathbb{R}^{2}\right)$. Recall that for an $n \in \mathbb{N}, W^{n, q}$ is defined as the completion of $C_{0}^{\infty}(\overline{\mathscr{C}} ; \mathbb{R})$, or $C_{0}^{\infty}\left(\overline{\mathscr{C}} ; \mathbb{R}^{2}\right)$ with respect to the norm

$$
\|v\|_{W^{n, q}}=\left(\sum_{|\alpha| \leq n} \int_{\mathscr{O}}\left|D^{\alpha} v(x)\right|^{q} \mathrm{~d} x\right)^{1 / q} .
$$

Then for $r=\theta n+(n+1)(1-\theta), W^{r, q}$ is defined as the complex interpolation space $\left[W^{n, q}, W^{n+1, q}\right]_{\theta}$. Finally, for $r>0$ we set $W^{-r, q}=\left(W^{-r, q^{*}}\right)^{*}$ where $q^{*}=$ $q /(q-1)$, and we identify $\left(L^{q}\right)^{*}$ with $L^{q^{*}}$. Clearly, $L^{q}=W^{0, q}$. We write for brevity $|\cdot|_{q}$ instead of $\|\cdot\|_{W^{0, q}}$, and $|\cdot|$ instead of $\|\cdot\|_{W^{0,2}}$, or $|\cdot|_{2}$.

For a proof of the following lemma, see [29].

LEMMA 3.1. There is a constant $C$ such that $|\nabla v|_{q} \leq C|\operatorname{curl} v|_{q}$ for every $v \in H^{1, q}$.

From now on $q \in(1, \infty)$. As before $\mathbb{X}_{q}$ denotes the closure of $C_{\mathrm{sol}}^{\infty}$ in $L^{q}$. For $u \in L^{q}$ we set

$$
\mathscr{P}_{q} u=u-\nabla p
$$


where $p$ is a solution to the following boundary value problem

$$
\Delta p=\operatorname{div} u \text { in } \mathscr{O}, \quad \frac{\partial p}{\partial \mathbf{n}}=\langle u, \mathbf{n}\rangle \quad \text { on } \partial \mathscr{O} .
$$

REMARK 3.1. Although $p$ is not unique, it is unique up to a constant in a simply connected domain. Thus $\nabla p$ is uniquely determined by $u$.

The following result is due to Fujiwara-Morimoto [23]. The case of $\mathscr{O}=\mathbb{R}^{2}$ is treated in [29].

LEMMA 3.2. $\quad \mathscr{P}_{q}$ is a bounded linear projection in $L^{q}$ and its range is equal to $\mathbb{X}_{q}$.

Consider the following modified Stokes operator:

$$
\left\{\begin{array}{l}
A_{q} u=-\mathscr{P}_{q} \Delta u, u \in \operatorname{Dom}\left(A_{q}\right), \\
\operatorname{Dom}\left(A_{q}\right)=\mathbb{X}_{q} \cap\left\{u \in W^{2, q}: \operatorname{curl} u=0 \text { on } \partial \mathscr{O}\right\} .
\end{array}\right.
$$

The following theorem gathers the analytical properties of $A_{q}$ which are needed in the proofs of the main existence results. Its proof is however postponed till Appendix A.

THEOREM 3.1. $\quad$ (i) The operator $-A_{q}$ is the generator of an analytic semigroup in the Banach space $\mathbb{X}_{q}$.

(ii) If $r>\frac{1}{2}+\frac{1}{2 q}$, then $\operatorname{Dom}\left(A_{q}^{r}\right)=\mathbb{X}_{q} \cap\left\{u \in W^{2 r, q}\right.$ : curl $u=0$ on $\left.\partial \mathscr{O}\right\}$. If $0<r<\frac{1}{2}+\frac{1}{2 q}$, then $\operatorname{Dom}\left(A_{q}^{r}\right)=\mathbb{X}_{q} \cap W^{2 r, q}$.

(iii) Let $r \in[0, \infty)$, and let us define the Sobolev space $H^{r, q}$ as the domain of $A_{q}^{r / 2}$ equipped with the graph norm. Then $\mathscr{P}_{q}$ is a bounded projection from $W^{r, q}$ into $H^{r, q}$.

(iv) Let $q^{*}$ be conjugate to $q$. Then there is a constant $C$ such that for $v \in$ $\operatorname{Dom}\left(A_{q}\right), u \in H^{1, q^{*}}=\operatorname{Dom}\left(A_{q^{*}}^{1 / 2}\right)$ one has $\left|\left\langle A_{q} v, u\right\rangle\right| \leq C\|v\|_{H^{1, q}}\|u\|_{H^{1, q^{*}}}$.

Note that from Theorem 3.1(ii), $H^{r, q}$ is a closed subspace of $W^{r, q}$. Thus, in particular the norm on $H^{r, q}$ and the norm induced from $W^{r, q}$ are equivalent. We set $H^{-r, q}=\left(H^{r, q^{*}}\right)^{*}$. Note that $\mathscr{P}_{q} \psi=\mathscr{P}_{r} \psi$ for all $q, r \in(1, \infty)$ and $\psi \in L^{q} \cap L^{r}$. Thus $A_{q} \psi=A_{r} \psi$ for $\psi \in \operatorname{Dom}\left(A_{q}\right) \cap \operatorname{Dom}\left(A_{r}\right)$. To simplify notation we shall write $\mathscr{P}$ and $A$ instead of $\mathscr{P}_{q}$ and $A_{q}$.

The remainder of this section is devoted to the study of the bilinear term in the Euler and Navier-Stokes equations. For $u, v \in C_{\text {sol }}^{\infty}$ write

$$
B(u, v)=-\mathscr{P}\left(\operatorname{div}\left(u v_{1}\right), \operatorname{div}\left(u v_{2}\right)\right) .
$$

Note that $B(u, v)=-\mathscr{P}(u, \nabla) v$. The theorem below gathers main properties of $B$. Its proof can be found in Appendix A. 
Theorem 3.2. Let $q \in[2, \infty)$, and let $B$ be given by (3.2). Then:

(i) The mapping $B$ has a unique extension to a continuous bilinear operator acting from $H^{2, q} \times H^{2, q}$ into $\mathbb{X}_{q}$. Moreover, there exists a constant $C$ such that for all $u, v \in H^{2, q}$ one has $|B(u, v)|_{q} \leq C\left(\|u\|_{H^{1, q}}\|v\|_{H^{2, q}}+\|u\|_{H^{2, q}}\|v\|_{H^{1, q}}\right)$.

(ii) For any $r>0, B$ has a unique extension to a continuous bilinear mapping acting from $H^{1, q} \times H^{1, q}$ into $H^{-r, q}$. In particular, there exists a constant $C$ such that

$$
\|B(u, v)\|_{H^{-r, q}} \leq C\|u\|_{H^{1, q}}\|v\|_{H^{1, q}} \quad \text { for all } u, v \in H^{1, q} .
$$

(iii) If $q>2$ then there exists a constant $C$ such that $|B(u, v)|_{q} \leq C\|u\|_{H^{1, q}}$ $\|v\|_{H^{1, q}}$ for all $u, v \in H^{1, q}$, and $|B(u, v)| \leq C\|u\|_{H^{1, q}}\|v\|_{H^{1,2}}$ for all $u, v \in H^{1,2} \cap$ $H^{1, q}$.

(iv) For every $u, v \in H^{1, q},\langle B(u, v), v\rangle=0$.

(v) For all $u, v \in H^{2, q},\left\langle\operatorname{curl} B(u, v)\right.$, curl $\left.v|\operatorname{curl} v|^{q-2}\right\rangle=0$.

\section{Probabilistic preliminaries.}

Definition 4.1. Let $\mathscr{\Re}=\left(\Omega, \mathscr{T},\left(\mathscr{T}_{t}\right)_{t \in[0, T]}, \mathbb{P}\right)$ be a filtered probability space, and let $\mathscr{H}$ be a real separable Hilbert space. By an $\left(\mathscr{T}_{t}\right)$-adapted cylindrical Wiener process on $\mathscr{H}$ we understand a family $W(t), t \geq 0$ of bounded linear operators from $\mathscr{H}$ into $L^{2}(\Omega, \mathscr{T}, \mathbb{P})$ such that:

(i) for all $t \geq 0$, and $\psi, \varphi \in \mathscr{H}, \mathbb{E} W(t) \psi W(t) \varphi=t\langle\psi, \varphi\rangle_{\mathscr{H}}$;

(ii) for each $\psi \in \mathscr{H}, W(t) \psi, t \geq 0$ is a real valued $\left(\mathscr{T}_{t}\right)$-adapted Wiener process.

REMARK 4.1. Assume that $E$ is a Banach space and $W(t), t \geq 0$ is a $E$ valued Wiener process defined on a filtered probability space $\Omega$. Replacing if necessary $E$ by its closed subspace we can assume that $E$ is equal to the support of the law $\mathscr{L}(W(1))$ of $W(1)$. Then (see, e.g., [28]), there is a unique densely and continuously imbedded into $E$ separable Hilbert space $\mathscr{H}$ such that

$$
\mathbb{E}(W(t), \psi)_{E, E^{*}}(W(s), \varphi)_{E, E^{*}}=t \wedge s\langle\psi, \varphi\rangle_{\mathscr{H}} \quad \text { for } t, s \geq 0, \psi, \varphi \in E^{*},
$$

where $(\cdot, \cdot)_{E, E^{*}}$ stands for the canonical bilinear form on $E \times E^{*}$, and we identify $(\mathscr{H})^{*}$ with $\mathscr{H}$, and then $E^{*}$ with a properly chosen subspace of $\mathscr{H}$. Thus, as $E^{*}$ is dense in $\mathscr{H}$, for any $t \geq 0$ the mapping

$$
E^{*} \ni \psi \mapsto(W(t), \psi)_{E, E^{*}} \in L^{2}(\Omega, \mathscr{F}, \mathbb{P})
$$

has the unique continuous extension to $\mathscr{H}$. We denote this extension also by $W(t)$. Note that $W$ is a cylindrical Wiener process on $\mathscr{H}$. The space $\mathscr{H}$ is called the reproducing kernel Hilbert space, shortly RKHS, or Cameron-Martin space of $W$.

REMARK 4.2. A cylindrical Wiener process on $\mathscr{H}$ can be viewed as a formal series

$$
W(t)=\sum_{k} W_{k}(t) f_{k}, \quad t \in[0, T],
$$


where $\left\{W_{k}\right\}$ is a sequence of independent standard 1-dimensional Wiener processes, and $\left\{f_{k}\right\}$ is an orthonormal basis of $\mathscr{H}$. This series does not converge in $\mathscr{H}$ if $\operatorname{dim} \mathscr{H}<\infty$. It does however in any Hilbert space $E$ such that the imbedding $\mathscr{H} \hookrightarrow E$ is Hilbert-Schmidt; see Remark 4.4. In this case $W$ is an $E$-valued Wiener process, and $\mathscr{H}$ is its RKHS.

EXAMPLE 4.1. Let $\mathscr{W}=\left(\mathscr{W}_{1}, \ldots, \mathscr{W}_{m}\right)$ be an $\mathbb{R}^{m}$-valued spatially homogeneous Wiener random field on $[0, T] \times \mathbb{R}^{2}$; see Definition 2.3. Note that $\mathscr{W}$ is a Wiener process on any weighted space $L^{2}\left(\mathbb{R}^{2}, \mathrm{e}^{-\rho|x|} \mathrm{d} x ; \mathbb{R}^{m}\right), \rho>0$. For a complex valued function $\psi$ on $\mathbb{R}^{2}$ we write

$$
\psi_{(\mathrm{s})}(x)=\overline{\psi(-x)}, \quad x \in \mathbb{R}^{2} .
$$

Let $\mu=\left(\mu_{1}, \ldots, \mu_{m}\right)$ be the spectral measure of $\mathscr{W}$. Let us denote by $L_{(\mathrm{s})}^{2}\left(\mathbb{R}^{2}, \mu_{j}\right)$ the closed subspace of $L^{2}\left(\mathbb{R}^{2}, \mu_{j} ; \mathbb{C}\right)$ of functions $\psi$ satisfying $\psi_{(\mathrm{s})}=\psi, \mu_{j}$-a.s. Finally let

$$
\mathscr{H}_{\mathscr{W}} \stackrel{\text { def }}{=}\left\{\left({\widehat{\psi} \mu_{1}}_{1}, \ldots, \widehat{\psi}_{m} \widehat{\mu}_{m}\right): \psi_{j} \in L_{(\mathrm{s})}^{2}\left(\mathbb{R}^{2}, \mu_{j}\right), j=1, \ldots, m\right\},
$$

where $\widehat{\psi}_{j}{ }_{j}$ is the Fourier transform of a tempered distribution $\psi_{j} \mu_{j}$. Then $\mathscr{H}_{\mathscr{W}}$ endowed with the norm

$$
\left\langle\left(\widehat{\psi_{1} \mu_{1}}, \ldots, \widehat{\psi_{m} \mu_{m}}\right),\left.\left(\widehat{\varphi_{1} \mu_{1}}, \ldots, \widehat{\varphi_{m} \mu_{m}}\right)\right|_{\mathscr{H} \mathscr{C}_{y}} \stackrel{\text { def }}{=} \sum_{j=1}^{m} \int_{\mathbb{R}^{2}} \psi_{j} \overline{\varphi_{j}} \mathrm{~d} \mu_{j}\right.
$$

is the RKHS of $\mathscr{W}$; see [38], Proposition 1.2.

EXAMPLE 4.2. Let $W$ be an $L^{2}\left(\mathscr{O} ; \mathbb{R}^{m}\right)$-valued Wiener process, and let $Q$ be its covariance operator. Note that in fact $W$ takes values in a smaller subspace of $L^{2}\left(\mathscr{O} ; \mathbb{R}^{m}\right)$, namely in $\left(\operatorname{Ker} Q^{1 / 2}\right)^{\perp}$. Let us equip $\mathscr{\mathscr { C }} \stackrel{\text { def }}{=}$ Range $Q^{1 / 2}$ with the scalar product

$$
\langle\psi, \varphi\rangle_{\mathscr{H}}=\langle u, v\rangle_{L^{2}\left(\mathscr{C}, \mathrm{d} x ; \mathbb{R}^{m}\right)}, \quad \text { where } \psi=Q^{1 / 2} u, \varphi=Q^{1 / 2} v, u, v \in\left(\operatorname{Ker} Q^{1 / 2}\right)^{\perp} .
$$

Then (see, e.g., [19]), $\mathscr{H}$ is the RKHS of $W$. Let $\left\{e_{k}\right\}$ be an orthonormal basis of $L^{2}\left(\mathscr{O} ; \mathbb{R}^{m}\right)$ consisting of the normalized eigenvectors of $Q^{1 / 2}$, and let $\left\{\lambda_{k}\right\}$ be the corresponding sequence of eigenvalues of $Q^{1 / 2}$. Then it is easy to see that $\left\{\lambda_{k} e_{k}\right\}$ is an orthonormal basis of $\mathscr{H}$. Thus using representation (4.1) we obtain

$$
W(t)=\sum_{k} \lambda_{k} W_{k}(t) e_{k}, \quad t \in[0, T]
$$

the series being convergent in $L^{2}\left(\Omega, \mathscr{F}, \mathbb{P} ; L^{2}\left(\mathscr{O} ; \mathbb{R}^{m}\right)\right)$.

Let $\mathscr{H}$ be a real separable Hilbert space, let $\left\{e_{k}\right\}$ be an orthonormal basis of $\mathscr{H}$, and let $\left\{\beta_{k}\right\}$ be a system of independent normal real-valued random variables defined on a probability space $(\Omega, \mathscr{T}, \mathbb{P})$. 
DEFINITION 4.2. Let $E$ be a real Banach space. A linear bounded operator $K: \mathscr{H} \rightarrow E$ is called $\gamma$-radonifying, or simply radonifying, iff the series $\sum_{k} \beta_{k} K e_{k}$ converges in $L^{2}(\Omega, \mathscr{F}, \mathbb{P} ; E)$.

Note that in view of the Itô-Nisio theorem (see [32]), $K: \mathscr{H} \rightarrow E$ is $\gamma$ radonifying iff the series $\sum_{k} \beta_{k} K e_{k}$ converges a.s. in $E$.

The set of all $\gamma$-radonifying operators from $\mathscr{H}$ into $E$ is denoted by $R(\mathscr{H}, E)$. Note that if $K \in R(\mathscr{H}, E)$ then $\sum_{k} \beta_{k} K e_{k}$ is a 0 -mean Gaussian $E$-valued random variable, and consequently Fernique's theorem yields that

$$
\|K\|_{R(\mathscr{H}, E)} \stackrel{\text { def }}{=}\left(\mathbb{E}\left|\sum_{k} \beta_{k} K e_{k}\right|_{E}^{2}\right)^{1 / 2}=\left(\int_{E}|e|_{E}^{2} \gamma_{K}(\mathrm{~d} e)\right)^{1 / 2}<\infty,
$$

where $\gamma_{K}$ denotes the law of the $E$-valued random vector $\sum_{k} \beta_{k} K e_{k}$. It is easy to see that for any $K \in R(\mathscr{H}, E),\|K\|_{R(H, E)}$ does not depend on the choice of $\left\{e_{k}\right\}$ and $\left\{\beta_{k}\right\}$. Moreover, $\|\cdot\|_{R(\mathscr{H}, E)}$ is a norm, and $\left(R(\mathscr{H}, E),\|\cdot\|_{R(\mathscr{\ell}, E)}\right)$ is a separable Banach space; for more details see [4] and [36].

REMARK 4.3. Let $W$ be a cylindrical Wiener process on $\mathscr{H}$, and let $E$ be a separable Banach space such that the imbedding $\mathscr{H} \hookrightarrow E$ is $\gamma$-radonifying. Let $\left\{e_{k}\right\}$ be an orthonormal basis of $\mathscr{H}$, and let $W_{k}(t)=W(t) e_{k}, k \in \mathbb{N}$ and $t \in[0, T]$. Then for every $t \in[0, T]$ the series $\sum_{k} W_{k}(t) e_{k}, t \in[0, T]$, converges in $L^{2}(\Omega, \mathscr{T}, \mathbb{P} ; E)$. Clearly, its limit, which we also denote by $W$, is a Wiener process on $E$ with the reproducing kernel Hilbert space $\mathscr{H}$, and $W$ does not depend on the choice of $\left\{e_{k}\right\}$.

REMARK 4.4. Assume that $E$ is a separable Hilbert space. Recall that a bounded linear operator $K$ from $\mathscr{H}$ into $E$ is called Hilbert-Schmidt iff

$$
\|K\|_{L_{(\mathrm{HS})}(\mathscr{H}, E)} \stackrel{\text { def }}{=}\left(\sum_{k=1}^{\infty}\left|K e_{k}\right|_{E}^{2}\right)^{1 / 2}<\infty
$$

for any orthonormal basis $\left\{e_{k}\right\}$ of $\mathscr{H}$. Let us denote by $L_{(\mathrm{HS})}(\mathscr{H}, E)$ the class of all Hilbert-Schmidt operators from $\mathscr{H}$ into $E$. Then, as a direct consequence of Theorem 4.1(iii) we have $R(\mathscr{H}, E)=L_{(\mathrm{HS})}(\mathscr{H}, E)$ and $\|\cdot\|_{R(\mathscr{H}, E)}=\|\cdot\|_{L_{(\mathrm{HS})}(\mathscr{H}, E)}$.

In what follows we fix a $q \in[2, \infty)$. The theorem below gives sufficient condition under which an integral $W^{r, q}$-valued operator $K$ is radonifying. The Sobolev space $W^{r, q}(\mathscr{O} ; \mathscr{H})$ appearing in its formulation can be defined in the standard way, see Section 3. The proof of Theorem 4.1 is given in Appendix B.

THEOREM 4.1. $\quad$ Let $K$ be a bounded linear operator acting from a real separable Hilbert space $\mathscr{H}$ into $W^{r, q}$, where $r \in[0, \infty)$. Assume that $K$ is given by the formula

$$
(K \psi)(x)=\langle\mathscr{K}(x), \psi\rangle_{\mathscr{H}} \quad \text { for } x \in \mathscr{O} \text { and } \psi \in \mathscr{H},
$$


where $\mathscr{K} \in W^{r, q}(\mathscr{O} ; \mathscr{H})$. Then $K \in R\left(\mathscr{H}, W^{r, q}\right)$ and there is an independent of $K$ constant $C$ such that $\|K\|_{R\left(\mathscr{H}, W^{r, q}\right)} \leq C\|\mathscr{K}\|_{W^{r, q}(\mathscr{O} ; \mathscr{C})}$.

Let $u=\left(u_{1}, \ldots, u_{m}\right)$ be an $\mathbb{R}^{m}$-valued function. In the proofs of Theorems 2.2 and 2.3 we apply Theorem 4.1 with $\mathscr{H}$ being the RKHS of an $\mathbb{R}^{m}$-valued spatially homogeneous Wiener random field, or an $L^{2}\left(\mathscr{O} ; \mathbb{R}^{m}\right)$-valued Wiener process respectively, see Lemmas 7.1 and 8.1. In both cases $K(u)$ is a linear operator on $\mathscr{H}$ given by

$$
K(u) \psi=\sum_{j=1}^{m} u_{j} \psi_{j}
$$

The remaining part of the present section is devoted to the construction and properties of the Itô integral in $L^{q}$ and $W^{1, q}$ spaces. In what follows $\mathscr{R}$ is a filtered probability space, and $W$ is an $\left(\mathscr{T}_{t}\right)$-adapted cylindrical Wiener process on a real separable Hilbert space $H$.

Let $V$ be a Banach space, and let us denote by $\mathscr{L}^{p}(0, T ; V)$ the Banach space of all $\left(\mathscr{F}_{t}\right)$-predictable $V$-valued processes $\sigma$ such that

$$
|\sigma|_{\mathscr{\ell}^{p}(0, T ; E)} \stackrel{\text { def }}{=}\left(\mathbb{E} \int_{0}^{T}|\sigma(t)|_{V}^{p} \mathrm{~d} t\right)^{1 / p}<\infty .
$$

Let us fix an orthonormal basis $\left\{e_{k}\right\}$ of $\mathscr{H}$, and let us denote by $\Pi_{n}$ the orthogonal projection onto the space spanned by $e_{1}, \ldots, e_{n}$. Let $E$ be a real separable Banach space, and let $\mathscr{L}_{0}(E)$ denote the class of all $\sigma \in \mathscr{L}^{2}(0, T ; R(\mathscr{H}, E))$ such that

$$
\sigma(\omega, t)=\sum_{j=1}^{n} \sigma_{j}(\omega) \Pi_{i} \chi_{\left(t_{j}, t_{j+1}\right]}
$$

for some $n, i \in \mathbb{N}, 0 \leq t_{1}<\cdots<t_{m+1} \leq T$ and $\sigma_{j} \in L^{2}\left(\Omega, \mathscr{T}_{t_{j}}, \mathbb{P} ; R(\mathscr{H}, E)\right)$. For $\sigma \in \mathscr{L}_{0}(E)$ and $t \in[0, T]$ we put

$$
\mathscr{I}_{t}^{W}(\sigma):=\sum_{j=1}^{n} \sum_{k=1}^{i}\left(W\left(t_{j+1} \wedge t\right)-W\left(t_{j} \wedge t\right)\right) e_{k} \sigma_{j} e_{k}
$$

In general $\mathscr{I}^{W}$ cannot be extended continuously to the whole $\mathscr{L}^{2}(0, T$; $R(H, E)$ ). This holds true for $E$ being an $M$-type 2 Banach space; see, e.g., [9] or [22]. Further to make the theory of stochastic integrals applicable we need a Burkholder type inequality. It turns out that this is also true when $E$ is an $M$-type 2 Banach space; see [9] and [22]. Since $L^{q}$ and $W^{r, q}$ are $M$-type 2 Banach spaces (see [10]), we have the following consequence of Theorems 2.4 and 3.3 from [22], see also [36] and [10].

THEOREM 4.2. Let $r \in[0, \infty)$. Then $\mathscr{L}_{0}\left(W^{r, q}\right)$ is dense in $\mathscr{L}^{2}(0, T$; $R\left(\mathscr{H}, W^{r, q}\right)$ ) and for each $t \in[0, T]$ there exists a unique extension of $\mathscr{I}_{t}^{W}$ to a linear bounded operator from $\mathscr{L}^{2}\left(0, T ; R\left(\mathscr{H}, W^{r, q}\right)\right)$ into $L^{2}\left(\Omega, \mathscr{T}_{t}, \mathbb{P} ; W^{r, q}\right)$. 
Moreover, there exists a constant $C$ such that for any $\sigma \in \mathscr{L}^{2}\left(0, T ; R\left(\mathscr{H}, W^{r, q}\right)\right)$ one has

$$
\mathbb{E} \sup _{s \in[0, t]}\left|\mathscr{I}_{t}^{W}(\sigma)\right|_{E}^{2} \leq C \mathbb{E} \int_{0}^{T}\|\sigma(s)\|_{R(\mathscr{H}, E)}^{2} \mathrm{~d} s, \quad t \in[0, T] .
$$

We denote the value of the extension of $\mathscr{I}_{t}^{W}$ on $\sigma \in \mathscr{L}^{2}\left(0, T ; R\left(\mathscr{H}, W^{r, q}\right)\right)$ by $\int_{0}^{t} \sigma(s) \mathrm{d} W(s)$ or by $\mathscr{I}_{t}^{W}(\sigma)$. Then, see [10] or [36], for any $\sigma, \mathscr{I}_{t}^{W}(\sigma), t \in[0, T]$, is a $W^{r, q}$-valued square integrable martingale with continuous modification.

For further references we recall Itô's formula for an $L^{q}$-valued process and real valued function

$$
\Psi_{q, p}(u)=|u|_{q}^{p}, \quad u \in L^{q} .
$$

For its proof we refer the reader to Appendix A from [13]; see also [36]. Before formulating the theorem we need to introduce some notation. Let $\gamma$ be a standard Gaussian distribution on a Hilbert space $\mathscr{H}$, and let $E$ be a Banach space. Let $K \in R(\mathscr{H}, E)$, and let $\gamma_{K}=\gamma \circ K^{-1}$. For any Banach space $V$, and for any bounded bilinear map $L: E \times E \rightarrow V$ we define

$$
\operatorname{tr}_{K} L=\int_{E} L(x, x) \mathrm{d} \gamma_{K}(x) .
$$

Below, $\langle\cdot, \cdot\rangle$ denotes the duality form on $\left(L^{q}\right)^{*} \times L^{q}$.

THEOREM 4.3. Let $p \geq q$, and let $\Psi_{q, p}$ be given by (4.5). Assume that

$$
\xi(t)=\xi(0)+\int_{0}^{t} a(s) \mathrm{d} s+\int_{0}^{t} \sigma(s) \mathrm{d} W(s), \quad t \in[0, T],
$$

with $a \in \mathscr{L}^{1}\left(0, T ; L^{q}\right)$ and $\sigma \in \mathscr{L}^{2}\left(0, T ; R\left(\mathscr{H}, L^{q}\right)\right)$. Then for all $t \geq 0$,

$$
\begin{aligned}
|\xi(t)|_{q}^{p}= & |\xi(0)|_{q}^{p}+p \int_{0}^{t}|\xi(s)|_{q}^{p-q}\left\langle|\xi(s)|^{q-2} \xi(s), a(s)\right\rangle \mathrm{d} s \\
& +p \int_{0}^{t}|\xi(s)|_{q}^{p-q}\left\langle|\xi(s)|^{q-2} \xi(s), \sigma(s) \mathrm{d} W(s)\right\rangle \\
& +\frac{1}{2} \int_{0}^{t} \operatorname{tr}_{\sigma(s)} \Psi_{q, p}^{\prime \prime}(\xi(s)) \mathrm{d} s .
\end{aligned}
$$

Note that $\psi \rightarrow\left\langle|\xi(s)|^{q-2} \xi(s), \sigma(s) \psi\right\rangle$ belongs to the space $R(\mathscr{H}, \mathbb{R})$, so the Itô integral above is well defined.

REMARK 4.5. Note that $v_{1}, v_{2} \in L^{q}$ we have

$$
\begin{aligned}
\Psi_{q, p}^{\prime \prime}(u)\left(v_{1}, v_{2}\right)= & p(q-1)|u|_{q}^{p-q} \int_{\mathscr{O}}|u(x)|^{q-2} v_{1}(x) v_{2}(x) \mathrm{d} x \\
& +p(p-q)|u|_{q}^{p-2 q} \int_{\mathscr{O}}|u(x)|^{q-2} u(x) v_{1}(x) \mathrm{d} x \\
& \times \int_{\mathscr{C}}|u(x)|^{q-2} u(x) v_{2}(x) \mathrm{d} x .
\end{aligned}
$$


Hence we easily obtain that

$$
\operatorname{tr}_{\sigma} \Psi_{q, p}^{\prime \prime}(u) \leq p(p-1)|u|_{q}^{p-2}\|\sigma\|_{R\left(\mathscr{\ell}, L^{q}\right)}^{2} .
$$

5. Solutions to modified Navier-Stokes equations. Let $\left\{e_{k}\right\} \subset H^{2,2} \cap$ $H^{2, q}$ be an orthonormal basis of $H^{1,2}$. We assume that it is also a Schauder basis of $H^{1, q}$. Let $\Pi_{n}, \Pi^{(n)}$ be orthonormal projections of $H^{1,2}$ into the spaces linspan $\left\{e_{1}, \ldots, e_{n}\right\}$ and linspan $\left\{e_{n}\right\}=\mathbb{R} e_{n}$, respectively. Let $\hat{\Pi}^{(n)}: H^{1,2} \rightarrow \mathbb{R}$ be defined by $\hat{\Pi}^{(n)}(v) e_{n}=\Pi^{(n)}(v), v \in H^{1,2}$.

Note that there is a constant $C$ such that

$$
\left\|\Pi_{n} v\right\|_{H^{1, q}} \leq C\|v\|_{H^{1, q}} \quad \text { and } \quad\left\|\Pi^{(n)} v\right\|_{H^{1, q}} \leq C\|v\|_{H^{1, q}}
$$

for all $n$ and $v \in H^{1,2} \cap H^{1, q}$. Thus $\Pi_{n}$ and $\Pi^{(n)}$ can be treated as linear projections on $H^{1, q}$.

From now on, we assume that the assumptions of Theorem 2.1 are fulfilled. Let $\rho \in C_{0}^{\infty}(\mathbb{R})$ be a non-negative function with the support in $[0,1]$ and mean $\int_{\mathbb{R}} \rho(x) \mathrm{d} x=1$. Let also $\chi_{n}=\chi_{[-n, n]}$. Recall that for all $t \in[0, T]$, $v \in H^{1,2} \cap H^{1, q}$, and $\psi \in \mathscr{H}, F(t, v), G(t, v) \psi$ belong to $W^{1,2} \cap W^{1, q}$. For $t \in[0, T]$ and $v \in H^{1,2} \cap H^{1, q}$ define

$$
\begin{aligned}
F_{n}(t, v)= & n^{-n} \Pi_{n} \int_{\mathbb{R}^{n}} F\left(t, \sum_{i=1}^{n} x_{i} e_{i}\right) \chi_{n}\left(\left|\sum_{i=1}^{n} x_{i} e_{i}\right|_{H^{1,2} \cap H^{1, q}}\right) \\
& \times \rho\left(n\left(\hat{\Pi}^{(1)} v-x_{1}\right)\right) \cdots \rho\left(n\left(\hat{\Pi}^{(n)} v-x_{n}\right)\right) \mathrm{d} x
\end{aligned}
$$

and, for $\psi \in \mathscr{H}$,

$$
\begin{aligned}
{\left[G_{n}(t, v) \psi\right]=} & n^{-n} \hat{\Pi}_{n} \int_{\mathbb{R}^{n}}\left[G\left(t, \sum_{i=1}^{n} x_{i} e_{i}\right) \psi\right] \chi_{n}\left(\left|\sum_{i=1}^{n} x_{i} e_{i}\right|_{H^{1,2} \cap H^{1, q}}\right) \\
& \times \rho\left(n\left(\hat{\Pi}^{(1)} v-x_{1}\right)\right) \cdots \rho\left(n\left(\hat{\Pi}^{(n)} v-x_{n}\right)\right) \mathrm{d} x .
\end{aligned}
$$

Note that the functions $F_{n}(t, \cdot)$ and $G_{n}(t, \cdot)$ are bounded and globally Lipschitz from $H^{1,2}$ into $W^{1,2}$, and from $H^{1, q}$ into $W^{1, q}$ and, respectively from $H^{1,2}$ into $R\left(\mathscr{H}, W^{1,2}\right)$, and from $H^{1, q}$ into $R\left(\mathscr{H}, W^{1, q}\right)$ (with bounds possibly depending on $n$ ). Moreover, there are functions $\tilde{a}_{1} \in L^{1}(0, T)$ and $\tilde{a}_{2} \in L^{r}(0, T)$, 
$r>2$ such that all $n \in \mathbb{N}, t \in(0, T)$ and $v \in H^{1, q}$ one has

$$
\begin{aligned}
\|F(t, v)\|_{W^{1,2}} & \leq \tilde{a}_{1}(t)\left(1+\|v\|_{H^{1,2}}\right), \\
\|F(t, v)\|_{W^{1, q}} & \leq \tilde{a}_{1}(t)\left(1+\|v\|_{H^{1, q}}\right), \\
\left\|F_{n}(t, v)\right\|_{W^{1,2}} & \leq \tilde{a}_{1}(t)\left(1+\|v\|_{H^{1,2}}\right), \\
\left\|F_{n}(t, v)\right\|_{W^{1, q}} & \leq \tilde{a}_{1}(t)\left(1+\|v\|_{H^{1, q}}\right), \\
\|G(t, v)\|_{L_{(\mathrm{HS})}\left(\mathscr{H}, W^{1,2}\right)} & \leq \tilde{a}_{2}(t)\left(1+\|v\|_{H^{1,2}}\right), \\
\|G(t, v)\|_{R\left(\mathscr{H}, W^{1, q}\right)} & \leq \tilde{a}_{2}(t)\left(1+\|v\|_{H^{1, q}}\right), \\
\left\|G_{n}(t, v)\right\|_{L_{(\mathrm{HS})}\left(\mathscr{H}, W^{1,2}\right)} & \leq \tilde{a}_{2}(t)\left(1+\|v\|_{H^{1,2}}\right), \\
\left\|G_{n}(t, v)\right\|_{R\left(\mathscr{H}, W^{1, q}\right)} & \leq \tilde{a}_{2}(t)\left(1+\|v\|_{H^{1, q}}\right) .
\end{aligned}
$$

Let $u_{0}^{(n)}=\Pi_{n} u_{0}$. Recall that the projection $\mathscr{P}$, the Stokes operator $A$, and the bilinear term $B$ were introduced in Section 3. Let $\varphi_{n}: H^{1,2} \cap H^{1, q} \rightarrow$ $H^{1,2} \cap H^{1, q}$ be defined by

$$
\varphi_{n}(u):= \begin{cases}u, & \text { if }|u|_{H^{1,2} \cap H^{1, q}} \leq n, \\ n|u|_{H^{1,2} \cap H^{1, q}}^{-1} u, & \text { otherwise. }\end{cases}
$$

It is well known, but see also Appendix in [11], that $\varphi_{n}$ is bounded and globally Lipschitz. Define $B_{n}(v)=B\left(\varphi_{n}(u), u\right)$. Then it follows from Theorem 3.2(iii) that $B_{n}$ is a (globally) Lipschitz map from $H^{1,2} \cap H^{1, q}$ to $\mathbb{X}_{2} \cap \mathbb{X}_{q}$. For brevity we write $B(v)=B(v, v)$.

THEOREM 5.1. Let $u_{0} \in H^{1,2} \cap H^{1, q}$, and let $\nu>0$. Let $W$ be a cylindrical Wiener process on $\mathscr{H}$ defined on a filtered probability space $\mathfrak{\AA}$. Then for any $n$ there is a unique adapted and continuous $H^{1,2} \cap H^{1, q}$-valued global strong solution $u^{(\nu, n)}$ to (1.3) - (1.4), that is a process satisfying the following conditions:

(i) $u^{(\nu, n)} \in L^{p}\left(\Omega ; L^{2}\left(0, T ; H^{2,2} \cap H^{2, q}\right) \cap C\left([0, T] ; H^{1,2} \cap H^{1, q}\right)\right)$ for any $p \in$ $[1, \infty)$

(ii) for $t \in[0, T]$ one has

$$
\begin{aligned}
u^{(\nu, n)}(t)= & u_{0}^{(n)}+\int_{0}^{t}\left\{-\nu A u^{(\nu, n)}(s)+B\left(u^{(\nu, n)}(s)\right)+\mathscr{P} F_{n}\left(s, u^{(\nu, n)}(s)\right)\right\} \mathrm{d} s \\
& +\int_{0}^{t} \mathscr{P} G_{n}\left(s, u^{(\nu, n)}(s)\right) \mathrm{d} W(s) .
\end{aligned}
$$

Moreover, for any $p \in(1, \infty)$,

$$
\sup _{\nu \in(0,1]} \sup _{n \in \mathbb{N}} \mathbb{E}\left\{\sup _{t \in[0, T]}\left(\left\|u^{(\nu, n)}(t)\right\|_{H^{1,2}}^{p}+\left\|u^{(\nu, n)}(t)\right\|_{H^{1, q}}^{p}\right)\right\}<\infty .
$$

Note that $F_{n}, G_{n}$ and $B_{n}$ are globally Lipschitz continuous and $U_{0}^{n} \in H^{2,2} \cap$ $H^{2, q}$. Thus the existence and uniqueness of a (global) mild solution $u^{(\nu, n)}$ to 
(1.3) - (1.4) follows from Theorem 4.10 in [10]. It follows next from Lemma 4.5 in [9] that in fact it is a strong solution. For an alternative detailed exposition in the case of the full, not approximated equation in a bounded domain see [14]. Hence Theorem 5.1 will be proved as soon as we verify Lemmas 5.1 and 5.2 formulated below.

LEMMA 5.1. Let $u^{(\nu, n)}$ be a solution to (1.3)-(1.4). Then for any $p \in[1, \infty)$ there is a constant $C<\infty$ independent of $\nu \in(0,1]$ and $n$ such that

$$
\mathbb{E} \sup _{t \in[0, T]}\left\|u^{(\nu, n)}(t)\right\|_{H^{1,2}}^{p} \leq C .
$$

PROOF. Let us first present the main points of the proof. Let us fix temporarily $p \in[2, \infty), \nu \in(0,1]$, and $n$. We adopt the convention that constants $c_{i}$ and $C_{i}$ depends only on $u_{0}, T, \tilde{a}_{1}, \tilde{a}_{2}$ and $p$. Recall that $|\cdot|$ denotes the $L^{2}$-norm. Set

$$
\psi(t)=\mathbb{E} \sup _{s \in[0, t]}\left|u^{(\nu, n)}(s)\right|^{4 p} \quad \text { and } \quad \varphi(t)=\mathbb{E} \sup _{s \in[0, t]}\left\|u^{(\nu, n)}(s)\right\|_{H^{1,2}}^{4 p} .
$$

The first step will be to show that there is a constant $C_{1}$ such that for all $0 \leq \kappa \leq t \leq T$

$$
\psi(t) \leq C_{1}\left\{1+\psi(\kappa)+\varphi(t) \int_{\kappa}^{t}\left(1+\tilde{a}_{1}(s)+\tilde{a}_{2}^{2}(s)\right) \mathrm{d} s\right\} .
$$

In this step we will use first Itô's lemma to the function $H(v)=|v|^{2 p}$, and then the Burkholder inequality. We will employ the growth conditions on $F$ and $G$, and Theorem 3.2(iv). Next we set

$$
\eta(t, x)=\operatorname{curl} u^{(\nu, n)}(t, x) \quad \text { and } \quad \vartheta(t)=\mathbb{E} \sup _{s \in[0, t]}|\eta(s)|^{4 p} .
$$

Then $\eta$ is a mild solution to the following stochastic partial differential equation (subject to Dirichlet boundary conditions if $\mathscr{O} \neq \mathbb{R}^{2}$ )

$$
\begin{aligned}
\mathrm{d} \eta= & {\left[\nu \Delta \eta+\operatorname{curl}\left\{B_{n}\left(u^{(\nu, n)}\right)+F_{n}\left(t, u^{(\nu, n)}\right)\right\}\right] \mathrm{d} t } \\
& +\left(\operatorname{curl} G_{n}\left(t, u^{(\nu, n)}\right)\right) \mathrm{d} W \\
= & {\left[\nu \Delta \eta-\left\langle u^{(\nu, n)}, \nabla\right\rangle \eta+\operatorname{curl} F_{n}\left(t, u^{(\nu, n)}\right)\right] \mathrm{d} t } \\
& +\left(\operatorname{curl} G_{n}\left(t, u^{(\nu, n)}\right)\right) \mathrm{d} W, \\
\eta(t, \cdot)= & 0 \quad \text { on } \partial \mathscr{O} .
\end{aligned}
$$

By uniqueness, since (5.4) has a unique strong $L^{2}(\mathscr{O})$-valued solution, $\eta$ is also a strong $L^{2}(\mathscr{O})$-valued solution. Hence, by applying again the Itô formula, 
the Burkholder inequality, and using Theorem 3.2(v) we will arrive at the following estimate

$$
\vartheta(t) \leq C_{2}\left\{1+\vartheta(\kappa)+(\vartheta(t)+\varphi(t)) \int_{\kappa}^{t}\left(\tilde{a}_{1}(s)+\tilde{a}_{2}^{2}(s)\right) \mathrm{d} s\right\}
$$

valid for all $0 \leq \kappa \leq t \leq T$. Taking into account Lemma 3.1 we may find $C_{3}$ and $C_{4}$ such that

$$
C_{3}(\psi(t)+\vartheta(t)) \leq \varphi(t) \leq C_{4}(\psi(t)+\vartheta(t)) .
$$

Consequently, combining (5.3) with (5.5) we obtain

$$
\varphi(t) \leq C_{5}\left\{1+\varphi(\kappa)+\varphi(t) \int_{\kappa}^{t}\left(1+\tilde{a}_{1}(s)+\tilde{a}_{2}^{2}(s)\right) \mathrm{d} s\right\} .
$$

Let $0=t_{1}<\cdots<t_{l}=T$ be a partition of $[0, T]$ such that for every $i=$ $1, \ldots, l-1$,

$$
\int_{t_{i}}^{t_{i+1}}\left(1+\tilde{a}_{1}(s)+\tilde{a}_{2}^{2}(s)\right) \mathrm{d} s \leq \frac{1}{2} .
$$

Then from (5.6) we have

$$
\varphi\left(t_{i+1}\right) \leq 2 C_{5}\left(1+\varphi\left(t_{i}\right)\right), \quad i=1, \ldots, l-1,
$$

and consequently

$$
\mathbb{E} \sup _{t \in[0, T]}\left\|u^{(\nu, n)}(t)\right\|_{H^{1,2}}^{4 p}=\varphi(T) \leq \sum_{j=1}^{l-1}\left(2 C_{5}\right)^{j}+\left(2 C_{5}\right)^{l-1}\left\|u_{0}\right\|_{H^{1,2}}^{4 p},
$$

which gives the desired estimate.

Let us now present some more details on the derivation of (5.3) and (5.5). For $t \in[0, T]$ we write

$$
\begin{aligned}
& \Phi(t)=\nu \mathscr{P} \Delta u^{(\nu, n)}(t)+\mathscr{P} F_{n}\left(t, u^{(\nu, n)}(t)\right), \\
& \mathscr{G}(t)=\mathscr{P} G_{n}\left(t, u^{(\nu, n)}(t)\right) .
\end{aligned}
$$

Let $\kappa<t$. Since $u^{(\nu, n)}$ is a strong $\mathbb{X}_{2}$-valued solution to (5.1), using Theorems 3.1(iv) and 3.2(ii) and the Itô formula (see [37] or [41]), we infer that

$$
\begin{aligned}
\left|u^{(\nu, n)}(t)\right|^{2 p}= & \left|u^{(\nu, n)}(\kappa)\right|^{2 p} \\
& +\int_{\kappa}^{t}\left[\left\langle H^{\prime}\left(u^{(\nu, n)}(s)\right), \Phi(s)\right\rangle+\frac{1}{2} \operatorname{trace} H^{\prime \prime}\left(u^{(\nu, n)}(s)\right) \mathscr{G}(s) \mathscr{G}^{*}(s)\right] \mathrm{d} s \\
& +\int_{\kappa}^{t} H^{\prime}\left(u^{(\nu, n)}(s)\right) \mathscr{G}(s) \mathrm{d} W(s) \\
= & :\left|u^{(\nu, n)}(\kappa)\right|^{2 p}+\int_{\kappa}^{t} I(s) \mathrm{d} s+\int_{\kappa}^{t} H^{\prime}\left(u^{(\nu, n)}(s)\right) \mathscr{G}(s) \mathrm{d} W(s) .
\end{aligned}
$$


Using now Theorems 3.1(iv) and 3.2(iv), and the growth property of $F_{n}$ we obtain

$$
\begin{aligned}
& \left\langle H^{\prime}\left(u^{(\nu)}(s)\right), \Phi(s)\right\rangle \\
& \quad \leq 2 p\left|u^{(\nu, n)}(s)\right|^{2(p-1)}\left[c_{1} \nu\left\|u^{(\nu, n)}(s)\right\|_{H^{1,2}}^{2}+\left\langle u^{(\nu, n)}(s), F_{n}\left(s, u^{(\nu, n)}(s)\right)\right\rangle\right] \\
& \quad \leq c_{2}\left(\tilde{a}_{1}(t)+1\right)\left(1+\left\|u^{(\nu, n)}(s)\right\|_{H^{1,2}}^{2 p}\right) .
\end{aligned}
$$

For the second term in the deterministic integral we have

$$
\begin{aligned}
& \frac{1}{2} \operatorname{trace} H^{\prime \prime}\left(u^{(\nu, n)}(s)\right) \mathscr{G}(s)(\mathscr{G}(s))^{*} \\
& \quad=p\left|u^{(\nu, n)}(s)\right|^{2(p-2)}\left[\left|u^{(\nu, n)}(s)\right|^{2}\|\mathscr{G}(s)\|_{L_{(\mathrm{HS})}\left(\mathscr{C}, L^{2}\right)}^{2}+2(p-1)\left|(\mathscr{G}(s))^{*} u^{(\nu, n)}(s)\right|^{2}\right] \\
& \quad \leq c_{3}\left|u^{(\nu, n)}(s)\right|^{2 p-2}\left\|G_{n}\left(s, u^{(\nu, n)}(s)\right)\right\|_{L_{(\mathrm{HS})}\left(\mathscr{H}, W^{1,2}\right)}^{2} \\
& \quad \leq c_{4} \tilde{a}_{2}^{2}(s)\left(1+\left\|u^{(\nu, n)}(s)\right\|_{H^{1,2}}^{2 p}\right) .
\end{aligned}
$$

Thus

$$
|I(s)| \leq c_{5}\left(1+\tilde{a}_{1}(s)+\tilde{a}_{2}^{2}(s)\right)\left(1+\left\|u^{(\nu, n)}(s)\right\|_{H^{1,2}}^{2 p}\right)
$$

and consequently

$$
\begin{array}{r}
\psi(t) \leq c_{6}\left\{1+\psi(\kappa)+\varphi(t)\left(\int_{\kappa}^{t}\left(1+\tilde{a}_{1}(s)+\tilde{a}_{2}^{2}(s)\right) \mathrm{d} s\right)^{2}\right. \\
\left.+\mathbb{E} \sup _{s \in[\kappa, t]}\left|\int_{\kappa}^{s} H^{\prime}\left(u^{(\nu, n)}(r)\right) \mathscr{G}(r) \mathrm{d} W(r)\right|^{2}\right\} .
\end{array}
$$

Applying now the Burkholder inequality we easily obtain that

$$
\begin{aligned}
& \mathbb{E} \sup _{s \in[\kappa, t]}\left|\int_{\kappa}^{s} H^{\prime}\left(u^{(\nu, n)}(r)\right) \mathscr{G}(r) \mathrm{d} W(r)\right|^{2} \\
& \quad \leq c_{7} \mathbb{E} \int_{\kappa}^{t} \tilde{a}_{2}^{2}(r)\left(1+\left\|u^{(\nu, n)}(r)\right\|_{H^{1,2}}^{4 p}\right) \mathrm{d} r \\
& \quad \leq c_{7}(1+\varphi(t)) \int_{\kappa}^{t} \tilde{a}_{2}^{2}(r) \mathrm{d} r,
\end{aligned}
$$

which gives (5.3). We can show (5.5). For brevity write

$$
\begin{aligned}
& \Psi(t)=\nu \Delta \eta(t)+\operatorname{curl}\left\{B_{n}\left(u^{(\nu, n)}(t)\right)+F_{n}\left(t, u^{(\nu, n)}(t)\right)\right\}, \\
& \tilde{G}(t)=\operatorname{curl} G\left(t, u^{(\nu, n)}(t)\right) .
\end{aligned}
$$

As we observed before, the process $\eta(t), t \in[0, T]$, given by (5.4) is a strong $L^{2}(\mathscr{O})$-valued solution to (5.4). Hence we may apply the Itô formula to the 
function $H$ and the process $\eta(t)$ and obtain

$$
\begin{aligned}
|\eta(t)|^{2 p}= & |\eta(\kappa)|^{2 p} \\
& +\int_{\kappa}^{t}\left[\left\langle H^{\prime}(\eta(s)), \Psi(s)\right\rangle+\frac{1}{2} \operatorname{trace} H^{\prime \prime}(\eta(s)) \tilde{G}(s)(\tilde{G}(s))^{*}\right] \mathrm{d} s \\
& +\int_{\kappa}^{t} H^{\prime}(\eta(s)) \tilde{G}(s) \mathrm{d} W(s) .
\end{aligned}
$$

Using Theorems 3.1(iv) and 3.2(v) we obtain

$$
\begin{aligned}
\left\langle H^{\prime}(\eta(s)), \Psi(s)\right\rangle & =2 p|\eta(s)|^{2(p-1)}\left[-\nu|\nabla \eta(s)|^{2}+\left\langle\eta(s), \operatorname{curl} F_{n}\left(s, u^{(\nu, n)}(s)\right)\right\rangle\right] \\
& \leq c_{8}|\eta(s)|^{2 p-1} \| F_{n}\left(s, u^{(\nu, n)}(s) \|_{W^{1,2}}\right. \\
& \leq c_{8} \tilde{a}_{1}(s)|\eta(s)|^{2 p-1}\left(1+\left\|u^{(\nu, n)}(s)\right\|_{H^{1,2}}\right) \\
& \leq c_{9} \tilde{a}_{1}(s)\left(1+|\eta(s)|^{2 p}+\left\|u^{(\nu, n)}(s)\right\|_{H^{1,2}}^{2 p}\right) .
\end{aligned}
$$

We have

$$
\begin{aligned}
& \frac{1}{2} \operatorname{trace} H^{\prime \prime}(\eta(s)) \tilde{G}(s)(\tilde{G}(s))^{*} \\
& \quad=p|\eta(s)|^{2(p-2)}\left[|\eta(s)|_{2}^{2}\|\tilde{G}(s)\|_{L_{(\mathrm{HS})}^{2}\left(\mathscr{H}, L^{2}\right)}^{2}+2(p-1)\left|(\tilde{G}(s))^{*} \eta(s)\right|_{2}^{2}\right] \\
& \quad \leq c_{10}|\eta(s)|^{2 p-2}\left\|G_{n}\left(s, u^{(\nu, n)}(s)\right)\right\|_{L_{(\mathrm{HS})}\left(\mathscr{H}, W^{1,2}\right)}^{2 p} \\
& \quad \leq c_{11} \tilde{a}_{2}^{2}(s)\left(1+|\eta(s)|^{2 p}+\left\|u^{(\nu, n)}(s)\right\|_{H^{1,2}}^{2 p} .\right.
\end{aligned}
$$

Applying now the Burkholder inequality we obtain

$$
\begin{aligned}
& \mathbb{E} \sup _{s \in[\kappa, t]}\left|\int_{\kappa}^{s} H^{\prime}(\eta(r)) \tilde{G}(r) \mathrm{d} W(r)\right|^{2} \\
& \quad \leq c_{12} \mathbb{E} \int_{\kappa}^{t} \tilde{a}_{2}^{2}(r)\left(1+|\eta(r)|^{4 p}+\left\|u^{(\nu, n)}(r)\right\|_{H^{1,2}}^{4 p}\right) \mathrm{d} r .
\end{aligned}
$$

Combining these estimates we get (5.5).

In what follows we need estimate for solutions in the space $H^{1, q}$, where $q \in[2, \infty)$. We use the inequality

$$
\|v\|_{H^{1, q}} \leq C\left(\|v\|_{H^{1,2}}+|\operatorname{curl} v|_{q}\right),
$$

which is a consequence of Lemma 3.1 and the imbedding $W^{1,2} \hookrightarrow L^{q}$.

LEMma 5.2. Let $u^{(\nu, n)}$ be the solution to (1.3) - (1.4). Then for any $p \in$ $[1, \infty)$ there is a constant $C<\infty$ independent of $\nu \in(0,1]$ and $n$ such that

$$
\mathbb{E} \sup _{t \in[0, T]}\left\|u^{(\nu, n)}(t)\right\|_{H^{1, q}}^{p} \leq C .
$$


Proof. As in the proof of Lemma 5.1 we fix $\nu, n$ and $p$. Define

$$
\eta(t, x)=\operatorname{curl} u^{(\nu)}(t, x) \quad \text { and } \quad \theta(t)=\mathbb{E} \sup _{s \leq t}\left|\eta^{(\nu)}(s)\right|_{q}^{2 p}
$$

Recall, see the proof of Lemma 5.2, that $\eta$ satisfies (5.4), and that $\Psi$ and $\tilde{G}$ are given by (5.7). Assume that $q>2$. First note that $\eta$ is a both strong and mild solution to (5.4). In particular, it follows from Theorem 4.6 and Lemma 4.3 from [9], and from Theorem 4.10 from [10] that for any $p \in[2, \infty)$,

$$
\eta \in L^{2}\left(\Omega, \mathscr{F}, \mathbb{P} ; L^{2}\left(0, T ; H^{2, q}\right)\right) \cap L^{p}\left(\Omega, \mathscr{F}, \mathbb{P} ; L^{\infty}\left(0, T ; H^{1, q}\right)\right) .
$$

For the above deduction we use the uniqueness of a weak solution to (5.4) and the imbedding $H^{1, q} \hookrightarrow L^{\infty}, q>2$.

Let $p \in[q, \infty)$, and $0 \leq \kappa \leq t \leq T$. Then by (5.10) we may apply the Itô formula for $H(\eta)=|\eta|_{q}^{p}$, see Theorem 4.3 and Remark 4.5. Thus we have

$$
\begin{aligned}
|\eta(t)|_{q}^{p} \leq & |\eta(\kappa)|_{q}^{p}+p \int_{\kappa}^{t}|\eta(s)|_{q}^{p-q}\left\langle|\eta(s)|^{q-2} \eta(s), \Psi(s)\right\rangle \mathrm{d} s \\
& +p \int_{\kappa}^{t}|\eta(s)|_{q}^{p-q}\left\langle|\eta(s)|^{q-2} \eta(s), \tilde{G}(s) \mathrm{d} W(s)\right\rangle \\
& +\frac{p(p-1)}{2} \int_{\kappa}^{t}|\eta(s)|_{q}^{p-2}\|\tilde{G}(s)\|_{R\left(\mathscr{H}, L^{q}\right)}^{2} \mathrm{~d} s .
\end{aligned}
$$

Using Theorem 3.2(v) and the growth estimates for $G$ and $F$ we get

$$
\begin{aligned}
|\eta(t)|_{q}^{p} \leq & |\eta(\kappa)|_{q}^{p}+c_{1} \int_{\kappa}^{t}|\eta(s)|_{q}^{p-1} \tilde{a}_{1}(s)\left(1+\left\|u^{(\nu, n)}(s)\right\|_{H^{1, q}}\right) \mathrm{d} s \\
& +\int_{\kappa}^{t}|\eta(s)|_{q}^{p-2} \tilde{a}_{2}^{2}(s)\left(1+\left\|u^{(\nu, n)}(s)\right\|_{H^{1, q}}^{2}\right) \mathrm{d} s+I(t),
\end{aligned}
$$

where

$$
I(t)=p \int_{\kappa}^{t}|\eta(s)|_{q}^{p-q}\left\langle|\eta(s)|_{q}^{q-2} \eta(s), \tilde{G}(s) \mathrm{d} W(s)\right\rangle .
$$

Using Lemma 5.1 and (5.8) we easily obtain the following estimate

$$
|\eta(t)|_{q}^{p} \leq|\eta(\kappa)|_{q}^{p}+c_{2}+c_{3} \int_{\kappa}^{t}|\eta(s)|_{q}^{p}\left(\tilde{a}_{1}(s)+\tilde{a}_{2}^{2}(s)\right) \mathrm{d} s+I(t),
$$

where $c_{2}, c_{3}$ are independent of $\nu \in(0,1], n, \kappa$, and $t$, and $\sigma$. The Burkholder inequality, see Theorem 4.2 , yields

$$
\mathbb{E} \sup _{\kappa \leq s \leq t}|I(s)|^{2} \leq c_{4}(1+\theta(t)) \int_{\kappa}^{t} \tilde{a}_{2}^{2}(s) \mathrm{d} s .
$$

Summing up we have

$$
\theta(t) \leq C_{1}\left\{1+\theta(\kappa)+\theta(t) \int_{\kappa}^{t}\left(\tilde{a}_{1}(s)+\tilde{a}_{2}^{2}(s)\right) \mathrm{d} s\right\},
$$


where $C$ is independent of $\nu, n, \tau, t$, and $\sigma$. Thus taking a partition $0=t_{1}<$ $\cdots<t_{l}=T$ such that

$$
\int_{t_{i}}^{t_{i+1}}\left(\tilde{a}_{1}(s)+\tilde{a}_{2}^{2}(s)\right) \mathrm{d} s \leq \frac{1}{2}
$$

we get

$$
\mathbb{E} \sup _{t \in[0, T]}\left\|u^{(\nu, n)}(t)\right\|_{H^{1, q}}^{2 p} \leq C_{2}(1+\theta(T)) \leq C_{2}\left(1+\sum_{j=1}^{l-1}(2 C)^{j}+(2 C)^{l-1}\left\|\Pi_{n} u_{0}\right\|_{H^{1, q}}^{2 p}\right) .
$$

Since $\left\{e_{n}\right\}$ is a Schauder basis of $H^{1, q}$ we have

$$
\sup _{n}\left\|\Pi_{n} u_{0}\right\|_{H^{1, q}}<\infty
$$

and the proof is complete.

6. Proof of Theorem 2.1. For further references we recall the wellknown Dubinsky criterion for compactness. Its proof can be found in [47], Theorem 4.1, page 132 .

LEMMA 6.1. Let $E_{0}, E_{1}$ and $E$ be reflexive Banach spaces such that the imbeddings $E_{0} \hookrightarrow E \hookrightarrow E_{1}$ are continuous and the imbedding $E_{0} \hookrightarrow E$ is compact. Let $p \in(1, \infty)$ and let $\Gamma$ be a bounded set in $L^{p}\left(0, T ; E_{0}\right)$ consisting of equicontinuous functions in $C\left([0, T] ; E_{1}\right)$. Then $\Gamma$ is relatively compact in $L^{p}(0, T ; E)$ and $C\left([0, T] ; E_{1}\right)$.

Let $\vartheta \in C^{\infty}\left(\mathbb{R}^{2}\right)$ be a strictly positive even function equal to $\exp \{-|x|\}$ for $|x| \geq 1$, and let us denote by $L_{\vartheta}^{2}$ the weighted space $L^{2}\left(\mathscr{O}, \vartheta(x) \mathrm{d} x ; \mathbb{R}^{2}\right)$. Clearly, if $\mathscr{O}$ is bounded, then the spaces $L^{2}$ and $L_{\vartheta}^{2}$ are equivalent. For a proof of the lemma below we refer the reader to [17], Lemma 3.4(i).

LEMMA 6.2. The imbedding $W^{1,2} \hookrightarrow L_{\vartheta}^{2}$ is compact.

In this section, $u^{(\nu, n)}, \nu \in(0,1], n \in \mathbb{N}$, is an $H^{1,2} \cap H^{1, q}$-valued solution to the modified Navier-Stokes problem (1.3) - (1.4). We assume that each $u^{(\nu, n)}$ is defined on a filtered probability space $\mathscr{K}$, and satisfies (1.3) driven by a cylindrical Wiener process $W$. Let us denote by $\mathscr{L}\left(u^{(\nu, n)}\right)$ the law of $u^{(\nu, n)}$ on the space of trajectories $C\left([0, T] ; H^{1,2} \cap H^{1, q}\right)$. Let

$$
M^{(\nu, n)}(t):=\int_{0}^{t} \mathscr{P} G_{n}\left(s, u^{(\nu, n)}(s)\right) \mathrm{d} W(s), \quad t \in[0, T],
$$

and let $\mathscr{L}\left(M^{(\nu, n)}\right)$ be the law of $M^{(\nu, n)}$ on $C\left([0, T] ; H^{1,2} \cap H^{1, q}\right)$.

Lemma 6.3. The family $\mathscr{L}\left(M^{(\nu, n)}\right), \nu \in(0,1], n \in \mathbb{N}$ is tight in $C\left([0, T] ; L_{\vartheta}^{2}\right)$. 
Proof. Let $\varepsilon>0$. We have to find a relatively compact set $\Gamma$ in $C([0, T]$; $\left.L_{\vartheta}^{2}\right)$ such that $\mathbb{P}\left(M^{(\nu, n)} \in M\right) \geq 1-\varepsilon$ for all $\nu \in(0,1], n \in \mathbb{N}$. To do this we are going to use the Dubinsky criterion with $E_{0}=H^{1,2}$ and $E_{1}=E=L_{\vartheta}^{2}$. First note that there is an $\alpha \in(0,1)$ such that

$$
\int_{0}^{t}(t-s)^{2(\alpha-1)} \tilde{a}_{2}^{2}(s) \mathrm{d} s<\infty \quad \text { for } t \in[0, T] .
$$

Let $p>(1-\alpha)^{-1}$, and let

$$
\mathscr{J} \psi(t)=\int_{0}^{t}(t-s)^{-\alpha} \psi(s) \mathrm{d} s, \quad t \in[0, T], \quad \psi \in L^{p}\left(0, T ; H^{1,2}\right) .
$$

Finally, let

$$
Y^{(\nu, n)}(t):=\frac{\sin (1-\alpha) \pi}{\pi} \int_{0}^{t}(t-s)^{\alpha-1} \mathscr{P} G_{n}\left(s, u^{(\nu, n)}(s)\right) \mathrm{d} W(s), \quad t \in[0, T] .
$$

Note that for all $\nu$ and $n$, the process $Y^{(\nu, n)}$ is well defined in $H^{1,2}$. Moreover, using (5.2) and the growth property of $G_{n}$ one can easily show that

$$
\sup _{\nu \in(0,1]} \sup _{n} \mathbb{E} \int_{0}^{T}\left\|Y^{(\nu, n)}(t)\right\|_{H^{1,2}}^{p} \mathrm{~d} t<\infty .
$$

Then, see [19] or [20], $M^{(\nu, n)}=\mathscr{J} Y^{(\nu, n)}$ and the desired conclusion follows from Lemma 6.2 and the fact that $\mathscr{J}$ transforms bounded sets in $L^{p}\left(0, T ; H^{1,2}\right)$ into equicontinuous bounded sets in $C\left([0, T] ; H^{1,2}\right)$.

Let $H$ be a Hilbert space. We denote by $L_{1}(H, H)$ the space of nuclear operators from $H$ into $H$. Obviously, $\left\{M^{(\nu, n)}\right\}$ are square integrable continuous $L_{\vartheta}^{2}$-valued martingales. Thus for every $\nu$ and $n$ there is a unique process $\ll M^{(\nu, n)} \gg$ with continuous trajectories in $L_{1}\left(L_{\vartheta}^{2}, L_{\vartheta}^{2}\right)$ such that for all $\psi, \varphi \in$ $L_{\vartheta}^{2}$ the process

$$
\left\langle M^{(\nu, n)}(t), \psi\right\rangle_{L_{\vartheta}^{2}}\left\langle M^{(\nu, n)}(t), \varphi\right\rangle_{L_{\vartheta}^{2}}-\left\langle\ll M^{(\nu, n)} \gg(t) \psi, \varphi\right\rangle_{L_{\vartheta}^{2}}, \quad t \in[0, T]
$$

is a real-valued continuous martingale. We call $\ll M^{(\nu, n)} \gg$ the quadratic variation process of $M^{(\nu, n)}$. Note that

$$
\ll M^{(\nu, n)} \gg(t)=\int_{0}^{t} j_{H^{1,2}, L_{\vartheta}^{2}} \mathscr{P} G_{n}\left(s, u^{(\nu, n)}(s)\right)\left(j_{H^{1,2}, L_{\vartheta}^{2}} \mathscr{P} G_{n}\left(s, u^{(\nu, n)}(s)\right)\right)^{*} \mathrm{~d} s,
$$

where $j_{H^{1,2}, L_{\vartheta}^{2}}$ denotes the imbedding of $H^{1,2}$ into $L_{\vartheta}^{2}$. We have the following consequence of Lemma 6.3 and the Métivier-Nakao theorem; see e.g. [34] or [35].

COROLlary 6.1. The family $\left\{\mathscr{L}\left(\ll M^{(\nu, n)} \gg\right)\right\}$ of the laws of $\left\{\ll M^{(\nu, n)} \gg\right\}$ is tight in $C\left([0, T] ; L_{1}\left(L_{\vartheta}^{2}, L_{\vartheta}^{2}\right)\right)$.

The next lemma plays a crucial role in the proof of Theorem 2.1. 
LEMMA 6.4. The family $\mathscr{L}\left(u^{(\nu, n)}\right), \nu \in(0,1]$, and $n \in \mathbb{N}$ is tight in $L^{2}\left(0, T ; L_{\vartheta}^{2}\right)$.

PRoOF. Let $v^{(\nu, n)}=u^{(\nu, n)}-M^{(\nu, n)}$. By Lemma 6.3 it is enough to show that the laws $\mathscr{L}\left(v^{(\nu, n)}\right)$ are tight in $L^{2}\left(0, T ; L_{\vartheta}^{2}\right)$. Let us define the weighted Sobolev space $W_{-\vartheta}^{1,2}$ as the completion of $C_{0}^{\infty}\left(\overline{\mathscr{O}}, \mathbb{R}^{2}\right)$ with respect to the norm

$$
\|v\|_{W_{-\vartheta}^{1,2}}=\left(\int_{\mathscr{O}}\left(|v(x)|^{2}+|\nabla v(x)|^{2}\right) \mathrm{e}^{|x|} \mathrm{d} x\right)^{1 / 2} .
$$

Then $W_{-\vartheta}^{1,2} \hookrightarrow W^{1,2} \hookrightarrow L^{2}$, and $W_{-\vartheta}^{1,2} \hookrightarrow L^{2}\left(\mathscr{O}, \mathrm{e}^{|x|} \mathrm{d} x ; \mathbb{R}^{2}\right)$. Let $E_{1}=W_{\vartheta}^{-1,2}:=$ $\left(W_{-\vartheta}^{1,2}\right)^{*}$, where we identify the adjoint space throughout the identity $\left(L^{2}\right)^{*}=$ $L^{2}$. Then $W^{-1,2} \hookrightarrow E_{1}$ and $L_{\vartheta}^{2} \hookrightarrow E_{1}$, with dense imbeddings. We use Lemma 6.1, for $E=L_{\vartheta}^{2}, E_{0}=W^{1,2}$, and $E_{1}$ defined above. Note that by Lemma 6.2, $E_{0}$ is compactly imbedded into $E$. We have

$$
v^{(\nu, n)}(t)=\Pi_{n} u_{0}+\int_{0}^{t}\left\{-\nu A u^{(\nu, n)}(s)+B\left(u^{(\nu, n)}(s)\right)+\mathscr{P} F_{n}\left(s, u^{(\nu, n)}(s)\right)\right\} \mathrm{d} s .
$$

Let $\varepsilon>0$. Then using (5.2), and Theorems 3.1(iv) and 3.2(ii) one can construct a set $\Sigma$ of equicontinuous in $W_{\vartheta}^{-1,2}$ valued equicontinuous functions such that

$$
\inf _{\nu \in(0,1], n \in \mathbb{N}} \mathbb{P}\left(v^{(\nu, n)} \in \Sigma\right)>1-\varepsilon .
$$

Thus the desired conclusion follows from the fact that as a consequence of (5.2) we have

$$
\begin{aligned}
\frac{1}{2} \sup _{\nu \in(0,1], n \in \mathbb{N}} \mathbb{E} \int_{0}^{T}\left\|v^{(\nu, n)}(t)\right\|_{H^{1,2}}^{2} \mathrm{~d} t \\
\leq \sup _{\nu \in(0,1], n \in \mathbb{N}} \mathbb{E} \int_{0}^{T}\left\|u^{(\nu, n)}(t)\right\|_{H^{1,2}}^{2} \mathrm{~d} t \\
\quad+\sup _{\nu \in(0,1], n \in \mathbb{N}} \mathbb{E} \int_{0}^{T}\left\|M^{(\nu, n)}(t)\right\|_{H^{1,2}}^{2} \mathrm{~d} t<\infty .
\end{aligned}
$$

LEMMA 6.5. Let $r \in(1, \infty)$, and let $v^{(l)}, l \in \mathbb{N}$ be a sequence of processes with trajectories in $L^{\infty}\left(0, T ; W^{1, r}\right)$, such that, for a fixed $p \in[1, \infty)$,

$$
\sup _{l} \mathbb{E}\left|v^{(l)}\right|_{L^{\infty}\left(0, T ; W^{1, r}\right)}^{p}<\infty .
$$

If $\left|v^{(l)}(t)-v(t)\right|_{L^{r}} \rightarrow 0, \mathrm{~d} t \times \mathbb{P}$-almost surely, then the process $v$ has trajectories in $L^{\infty}\left(0, T ; W^{1, r}\right)$ and $\mathbb{E}|v|_{L^{\infty}\left(0, T ; W^{1, r}\right)}^{p}<\infty$.

PROOF. Let $\left\{e_{k}\right\}$ be an unconditional Schauder basis of the both spaces $L^{r}$ and $W^{1, r}$. Then each element $z \in L^{r}$ has the unique representation $z=\sum z_{j} e_{j}$. Moreover, the projections

$$
\Pi_{k} z \stackrel{\text { def }}{=} \sum_{j=1}^{k} z_{j} e_{j}, \quad k \in \mathbb{N},
$$


are uniformly bounded in both $L^{r}$ and $W^{1, r}$. In particular, $z \in L^{r}$ belongs to $W^{1, r}$ iff $\sup _{k}\left\|\Pi_{k} z\right\|_{W^{1, r}}<\infty$. If this is a case, then

$$
\|z\|_{W^{1, r}}=\lim _{k \rightarrow \infty}\left\|\Pi_{k} z\right\|_{W^{1, r}} .
$$

Thus, as $\left\|\Pi_{k}\right\|_{L\left(W^{1, r}, W^{1, r}\right)}, k \in \mathbb{N}$ is bounded, for $p, s \geq 2$ we have, using the Fatou lemma,

$$
\begin{aligned}
\mathbb{E}\left(\int_{0}^{T} \lim _{k \rightarrow \infty}\left\|\Pi_{k} v(t)\right\|_{W^{1, r}}^{s} \mathrm{~d} t\right)^{p / s} & \leq \liminf _{k \rightarrow \infty} \mathbb{E}\left(\int_{0}^{T}\left\|\Pi_{k} v(t)\right\|_{W^{1, r}}^{s} \mathrm{~d} t\right)^{p / s} \\
& \leq\left. T^{p / s} \liminf _{k \rightarrow \infty} \liminf _{l \rightarrow \infty}|\mathbb{E}| \Pi_{k} v^{(l)}\right|_{L^{\infty}\left(0, T ; W^{1, r}\right)} ^{p} \\
& \leq C T^{p / s} \sup _{l \in \mathbb{N}} \mathbb{E}\left|v^{(l)}\right|_{L^{\infty}\left(0, T ; W^{1, r}\right)}^{p}<\infty .
\end{aligned}
$$

Hence $v$ has trajectories in $W^{1, r}$ and

$$
\mathbb{E}|v|_{L^{\infty}\left(0, T ; W^{1, r}\right)}^{p}=\lim _{s \rightarrow \infty} \mathbb{E}|v|_{L^{s}\left(0, T ; W^{1, r}\right)}^{p} \leq C \sup _{l \in \mathbb{N}} \mathbb{E}\left|v^{(l)}\right|_{L^{\infty}\left(0, T ; W^{1, r}\right)}^{p}<\infty,
$$

which completes the proof.

Proof of Theorem 2.1. Let $\tilde{\mathscr{H}}$ be a Hilbert space such that $\mathscr{H} \hookrightarrow \tilde{\mathscr{H}}$ with a Hilbert-Schmidt imbedding, and let

$$
\mathscr{X}=L^{2}\left(0, T ; L_{\vartheta}^{2}\right) \times C\left([0, T] ; L_{\vartheta}^{2}\right) \times C\left([0, T] ; L_{1}\left(L_{\vartheta}^{2}, L_{\vartheta}^{2}\right)\right) \times C([0, T] ; \tilde{\mathscr{H}}) .
$$

Note that $W$ is then a process with continuous trajectories in $\tilde{\mathscr{H}}$. By Lemmas 5.3, 5.4 and Corollary 6.1, the family of laws $\mathscr{L}\left(u^{(\nu, n)}, M^{(\nu, n)}, \ll M^{(\nu, n)} \gg, W\right)$, $\nu \in(0,1], n \in \mathbb{N}$ of $\left(u^{(\nu, n)}, M^{(\nu, n)}, \ll M^{(\nu, n)} \gg, W\right)$ on $\mathscr{X}$ is tight, and hence by the Prokhorov theorem, it is relatively weakly compact. So there are sequences $\nu_{l} \downarrow 0$ and $n_{l} \uparrow \infty$ such that $\left(u^{\left(\nu_{l}, n_{l}\right)}, M^{\left(\nu_{l}, n_{l}\right)}, \ll M^{(\nu, n)} \gg, W\right)$ converges weakly as $l \uparrow \infty$.

By the Skorokhod imbedding theorem (see, e.g., [27]), there exist a probability space $\mathfrak{U}=\left(\Omega, \mathscr{F},\left(\mathscr{F}_{t}\right), \mathbb{P}\right)$, random elements in $\mathscr{X},(v, M, m, V)$ and $\left\{\left(v^{(l)}, M^{(l)}, m^{(l)}, V^{(l)}\right)\right\}, l \in \mathbb{N}$, defined on $\Omega$, such that the laws of $\left(u^{\left(\nu_{l}, n_{l}\right)}\right.$, $\left.M^{\left(\nu_{l}, n_{l}\right)}, \ll M^{\left(\nu_{l}, n_{l}\right)} \gg, W\right)$ and $\left(v^{(l)}, M^{(l)}, m^{(l)}, V^{(l)}\right)$ are the same, and $\left(v^{(l)}, M^{(l)}\right.$, $\left.m^{(l)}, V^{(l)}\right) \rightarrow(v, M, m, V), \mathbb{P}$-almost surely in $\mathscr{X}$. Thus, in particular,

$$
\lim _{l \rightarrow \infty} \int_{0}^{T}\left|v^{(l)}(t)-v(t)\right|_{L_{\vartheta}^{2}}^{2} \mathrm{~d} t=0, \quad \mathbb{P} \text {-a.s. }
$$

Note that for every $l, V^{(l)}$ is a cylindrical Wiener processes on $\mathscr{H}$, and $v^{(l)}$ is the solution to the appropriate Navier-Stokes equations driven by $V^{(l)}$. Moreover,

$$
M^{(l)}(t)=\int_{0}^{t} \mathscr{P} G_{n_{l}}\left(s, v^{(l)}(s)\right) \mathrm{d} V^{(l)}(s), \quad t \in[0, T]
$$

and

$$
\begin{aligned}
m^{(l)}(t) & =\ll M^{(l)} \gg(t) \\
& =\int_{0}^{t} j_{H^{1,2}, L_{\vartheta}^{2}} \mathscr{P} G_{n_{l}}\left(s, v^{(l)}(s)\right)\left(j_{H^{1,2}, L_{\vartheta}^{2}} \mathscr{P} G_{n_{l}}\left(s, v^{(l)}(s)\right)\right)^{*} \mathrm{~d} s .
\end{aligned}
$$


Let $p \geq 2$. Then as $H^{1,2} \hookrightarrow L_{\vartheta}^{2},(5.2)$ and the Fatous lemma yield

$$
\mathbb{E}\left(\int_{0}^{T}|v(t)|_{L_{\vartheta}^{2}}^{2} \mathrm{~d} t\right)^{p} \leq \liminf _{l \rightarrow \infty} \mathbb{E}\left(\int_{0}^{T}\left|v^{(l)}(s)\right|_{L_{\vartheta}^{2}}^{2} \mathrm{~d} t\right)^{p}<\infty .
$$

Thus for any $p \geq 2$ we have

$$
\sup _{l \in \mathbb{N}} \mathbb{E}\left(\int_{0}^{T}\left|v^{(l)}(t)-v(t)\right|_{L_{\vartheta}^{2}}^{2} \mathrm{~d} t\right)^{p}<\infty,
$$

and consequently, the sequence $\int_{0}^{T}\left|v^{(l)}(t)-v(t)\right|_{L_{\vartheta}^{2}}^{2} \mathrm{~d} t, l \in \mathbb{N}$ is uniformly integrable, and hence from (5.1) we have

$$
\lim _{l \rightarrow \infty} \mathbb{E} \int_{0}^{T}\left|v^{(l)}(t)-v(t)\right|_{L_{\vartheta}^{2}}^{2} \mathrm{~d} t=0 .
$$

Taking a subsequence we may assume that $v^{(l)}(t, x) \rightarrow v(t, x), \mathrm{d} t \times \mathrm{d} x \times \mathbb{P}$-a.s., and

$$
\lim _{l \rightarrow \infty} \int_{0}^{T}\left|v^{(l)}(t)-v(t)\right|_{L_{\vartheta}^{2}}^{2} \mathrm{~d} t=0, \quad \mathbb{P} \text {-a.s.. }
$$

Since $\left\{v^{(l)}\right\}$ is bounded in $L^{p}\left(\Omega, \mathscr{T}, \mathbb{P} ; L^{\infty}\left(0, T ; L^{r}\right)\right), r=2, q$, we may assume that $\left|v^{(l)}(t)-v(t)\right|_{L^{r}} \rightarrow 0, \mathrm{~d} t \times \mathbb{P}$-a.s. for $r=2, q$. Lemma 6.5 yields that $v$ has a trajectories from $L^{\infty}\left(0, T ; H^{1,2} \cap H^{1, q}\right)$ and that for every $p \geq 2$, one has

$$
\mathbb{E}\left\{\sup _{t \in[0, T]}\|v(t)\|_{H^{1,2}}^{p}+\sup _{t \in[0, T]}\|v(t)\|_{H^{1, q}}^{p}\right\}<\infty .
$$

The sequence $\left\{M^{(l)}\right\}$ converges $\mathbb{P}$-a.s. to $M$. Note that $M$ is a square integrable continuous $L_{\vartheta}^{2}$-valued martingale with quadratic variation $m$. Our goal is to show that

$$
\ll M \gg(t)=\int_{0}^{t} j_{H^{1,2}, L_{\vartheta}^{2}} \mathscr{P} G(s, v(s))\left(j_{H^{1,2}, L_{\vartheta}^{2}} \mathscr{P} G(s, v(s))\right)^{*} \mathrm{~d} s, \quad t \in[0, T] .
$$

To do this it is enough to prove that for all $t \in[0, T]$ and $z \in C_{0}^{\infty}\left(\overline{\mathscr{O}} ; \mathbb{R}^{2}\right)$ the sequence

$$
J_{l}=\int_{0}^{t}\left|\left(j_{H^{1,2}, L_{\vartheta}^{2}} \mathscr{P} G_{n_{l}}\left(s, v^{(l)}(s)\right)\right)^{*} z\right|_{\mathscr{H}}^{2} \mathrm{~d} s, \quad l \in \mathbb{N},
$$

converges in probability to

$$
J=\int_{0}^{t}\left|\left(j_{H^{1,2}, L_{\vartheta}^{2}} \mathscr{P} G(s, v(s))\right)^{*} z\right|_{\mathscr{H}}^{2} \mathrm{~d} s .
$$

Note that for all $\varepsilon>0$ and $R>0$ one has

$$
\begin{aligned}
& \mathbb{P}\left(\left|J_{l}-J\right|>\varepsilon\right) \leq \tilde{\mathbb{P}}\left(\left|J_{l}-J\right|>\varepsilon \text { and } \sup _{0 \leq s \leq T}\left|v^{(l)}(s)\right| \leq R\right) \\
&+\mathbb{P}\left(\sup _{0 \leq s \leq T}\left|v^{(l)}(s)\right|>R\right)
\end{aligned}
$$


Therefore the convergence in probability follows from the assumptions (i) and (ii) of Theorem 2.1, and (6.2), (6.3) and the Lebesgue dominated convergence theorem.

Using now the representation theorem ([19], Theorem 8.2) we can find a filtered probability space $\left(\Omega^{\prime}, \mathscr{F}^{\prime},\left(\mathscr{F}_{t}^{\prime}\right), \mathbb{P}^{\prime}\right)$, and a cylindrical Wiener process $\tilde{W}$ on $\mathscr{H}$, which is defined on the probability space

$$
\tilde{\mathscr{R}}=\left(\tilde{\Omega}=\Omega \times \Omega^{\prime}, \tilde{\mathscr{F}}=\mathscr{F} \times \mathscr{F}^{\prime},\left(\tilde{\mathscr{F}_{t}}\right)=\left(\mathscr{F}_{t} \times \mathscr{F}_{t}^{\prime}\right), \tilde{\mathbb{P}}=\mathbb{P} \times \mathbb{P}^{\prime}\right),
$$

such that the process $\mathscr{M}\left(t, \omega_{1}, \omega_{2}\right)=M\left(t, \omega_{1}\right)$ has the following form:

$$
\mathscr{M}\left(t, \omega_{1}, \omega_{2}\right)=\int_{0}^{t} \mathscr{P} G\left(s, u\left(s, \omega_{1}, \omega_{2}\right)\right) \mathrm{d} \tilde{W}\left(s, \omega_{1}, \omega_{2}\right),
$$

where $u\left(s, \omega_{1}, \omega_{2}\right)=v\left(s, \omega_{1}\right)$. Clearly, the process $u$ is adapted to the filtration $\left(\tilde{\mathscr{F}_{t}}\right)$, and by $(6.3)$, for every $p \in[2, \infty)$,

$$
\tilde{\mathbb{E}}\left\{\sup _{t \in[0, T]}\|u(t)\|_{H^{1,2}}^{p}+\sup _{t \in[0, T]}\|u(t)\|_{H^{1, q}}^{p}\right\}<\infty .
$$

The last task is to show that $u$ satisfies the integral equation in Definition 2.1. To do this we fix $t \in[0, T]$ and $z \in C_{0}^{\infty}\left(\mathscr{O} ; \mathbb{R}^{2}\right)$ satisfying $\operatorname{div} z=0$. We have already shown that, $\tilde{\mathbb{P}}$-a.s.,

$$
\begin{aligned}
\left\langle\int_{0}^{t} \mathscr{P} G_{n_{l}}\left(s, v^{(l)}(s)\right) \mathrm{d} W(s), z\right\rangle & =\left\langle M^{(l)}(t), z\right\rangle \rightarrow\langle M(t), z\rangle \\
& =\left\langle\int_{0}^{t} \mathscr{P} G(t, u(s)) \mathrm{d} \tilde{W}(s), z\right\rangle \\
& =\left\langle\int_{0}^{t} G(t, u(s)) \mathrm{d} \tilde{W}(s), z\right\rangle .
\end{aligned}
$$

For $t \in[0, T]$ and $z \in C_{\text {sol }}^{\infty}$ we define

$$
\begin{aligned}
I_{l} & =\int_{0}^{t}\left\langle\mathscr{P} F_{n_{l}}\left(s, v^{(l)}(s)\right), z\right\rangle \mathrm{d} s, \\
I & =\int_{0}^{t}\langle\mathscr{P} F(s, u(s)), z\rangle \mathrm{d} s=\int_{0}^{t}\langle F(s, u(s)), z\rangle \mathrm{d} s .
\end{aligned}
$$

We are showing that $I_{l}$ converges to $I$ in probability $\tilde{\mathbb{P}}$. Since for all $l, \varepsilon>0$, and $R>0$ we have

$$
\begin{aligned}
\tilde{\mathbb{P}}\left(\left|I_{l}-I\right|>\varepsilon\right) \leq \tilde{\mathbb{P}} & \left(\left|I_{l}-I\right|>\varepsilon \text { and } \sup _{0 \leq s \leq T}\left|v^{(l)}(s)\right| \leq R\right) \\
& +\tilde{\mathbb{P}}\left(\sup _{0 \leq s \leq T}\left|v^{(l)}(s)\right|>R\right)
\end{aligned}
$$

the convergence follows from the assumptions (i) and (ii) of Theorem 2.1, and (6.2), (6.3) and the Lebesgue dominated convergence theorem. 
Clearly $v^{(l)}(0) \rightarrow u_{0}$, and from (6.2) we have $\left\langle v^{(l)}(t), z\right\rangle \rightarrow\langle u(t), z\rangle$. Also from (6.2) we have

$$
\int_{0}^{t} \sum_{j=1}^{2}\left\langle v^{(l)}(s) v_{j}^{(l)}(s), \partial_{j} z\right\rangle \mathrm{d} s \rightarrow \int_{0}^{t} \sum_{j=1}^{2}\left\langle u(s) u_{j}(s), \partial_{j} z\right\rangle \mathrm{d} s .
$$

Finally,

$$
\nu_{l} \int_{0}^{t}\left\langle v^{(l)}(s), \Delta z\right\rangle \mathrm{d} s \rightarrow 0 .
$$

Thus $u$ satisfies the integral equation in Definition 2.1, and the proof is complete.

\section{Proof of Theorem 2.2.}

LEMMA 7.1. Let $\mathscr{W}$ be an $\mathbb{R}^{m}$-valued spatially homogeneous random Wiener field with the spectral measure $\mu=\left(\mu_{1}, \ldots, \mu_{m}\right)$, and let $\mathscr{H}_{\mathscr{W}}$ be its RKHS. Let $u=\left(u_{1}, \ldots, u_{m}\right) \in W^{1, q}\left(\mathbb{R}^{2} ; \mathbb{R}^{m}\right)$, and let $K(u)$ be given by (4.4). Assume that

$$
\int_{\mathbb{R}^{2}}\left(1+|y|^{q}\right) \mathrm{d} \mu_{j}(y)<\infty \quad \text { for } j=1, \ldots, m .
$$

Then $K(u) \in R\left(\mathscr{H}_{\mathscr{W}}, W^{1, q}\right)$, and there is an independent of $u$ constant $C$ such that

$$
\|K(u)\|_{R\left(\mathscr{H}_{\mathscr{Y}}, W^{1, q}\right)} \leq C\|u\|_{W^{1, q}\left(\mathbb{R}^{2} ; \mathbb{R}^{m}\right)}\left(\sum_{j=1}^{m} \int_{\mathbb{R}^{2}}\left(1+|y|^{q}\right) \mathrm{d} \mu_{j}(y)\right)^{1 / q} .
$$

Proof. Set

$$
L_{(\mathrm{s})}^{2}(\mu)=\left\{\psi=\left(\psi_{1}, \ldots, \psi_{m}\right): \psi_{j} \in L_{(\mathrm{s})}^{2}\left(\mathbb{R}^{2}, \mu_{j}\right)\right\},
$$

and let us equip $L_{(\mathrm{s})}^{2}(\mu)$ with the scalar product

$$
\langle\psi, \varphi\rangle_{L_{(\mathrm{s})}^{2}(\mu)} \stackrel{\text { def }}{=} \sum_{j=1}^{m} \int_{\mathbb{R}^{2}} \psi_{j} \overline{\varphi_{j}} \mathrm{~d} \mu_{j}
$$

Finally let

$$
j(\psi)=\left(\widehat{\psi}_{i} \mu_{1}, \ldots, \widehat{\psi_{m} \mu_{m}}\right), \quad \psi \in L_{(\mathrm{s})}^{2}(\mu) .
$$

Clearly $j$ is an isomorphism between $L_{(\mathrm{s})}^{2}(\mu)$ and $\mathscr{H}_{\mathscr{W}}$. Thus

$$
K(u) \in R\left(\mathscr{C}_{\mathscr{W}}, W^{1, q}\right) \Longleftrightarrow K(u) j \in R\left(L_{(\mathrm{s})}^{2}(\mu), W^{1, q}\right) .
$$


Now we have

$$
\begin{aligned}
(K(u) j)(\psi)(x) & =\sum_{j=1}^{m} u_{j}(x){\widehat{\psi_{j} \mu}}_{j}(x) \\
& =\sum_{j=1}^{m}(2 \pi)^{-1} \int_{\mathbb{R}^{2}} u_{j}(x) \exp \{i\langle x, y\rangle\} \psi_{j}(y) \mathrm{d} \mu_{j}(y) \\
& =\langle\mathscr{K}(u)(x), \psi\rangle_{L_{(\mathrm{s})}^{2}(\mu)},
\end{aligned}
$$

where

$$
\mathscr{K}(u)(x)=(2 \pi)^{-1}\left(u_{1}(x) \exp \{-i\langle x, \cdot\rangle\}, \ldots, u_{m}(x) \exp \{-i\langle x, \cdot\rangle\}\right) .
$$

Note that $\mathscr{K}(u) \in W^{1, q}\left(\mathbb{R}^{2} ; L_{(\mathrm{s})}^{2}(\mu)\right)$, and there is an independent of $u$ and $\mu$ constant $C_{1}$ such that

$$
\|\mathscr{K}(u)\|_{W^{1, q}\left(\mathbb{R}^{2} ; L_{(\mathrm{s})}^{2}(\mu)\right)} \leq C_{1}\|u\|_{W^{1, q}\left(\mathbb{R}^{2} ; \mathbb{R}^{m}\right)}\left(\sum_{j=1}^{m} \int_{\mathbb{R}^{2}}\left(1+|y|^{q}\right) \mathrm{d} \mu_{j}(y)\right)^{1 / q} .
$$

Hence, Theorem 4.1 gives the desired conclusion.

Proof OF TheOREM 2.2. We prove the theorem by showing that $F$ and $G$ given by (2.2) satisfy the hypothesis (i) and (ii) of Theorem 2.1. To do this take $v \in H^{1, q}$. Then $F(t, v)(x)=f(t, x, v(x))$. Clearly, $F(t, v) \in W^{1, q}$ and

$$
\begin{aligned}
\|F(t, v)\|_{W^{1, q}}^{q} & =\int_{\mathbb{R}^{2}}\left\{|f(t, x, v(x))|^{q}+\left|\frac{\mathrm{d}}{\mathrm{d} x} f(t, x, v(x))\right|^{q}\right\} \mathrm{d} x \\
& \leq C_{1} a_{1}^{q}(t) \int_{\mathbb{R}^{2}}\left\{|\phi(x)|^{q}+|v(x)|^{q}+|\nabla v(x)|^{q}\right\} \mathrm{d} x \\
& \leq C_{2} a_{1}^{q}(t)\left(1+\|v\|_{W^{1, q}}^{q}\right),
\end{aligned}
$$

where $\phi \in L^{q}$ and $a_{1} \in L^{1}(0, T)$. Thus $F$ satisfies (i). Finally, for $z \in C_{\text {sol }}^{\infty}$ and $u, v \in W^{1, q}$ we have

$$
\begin{aligned}
|\langle F(t, v)-F(t, u), z\rangle| & =\left|\int_{\mathbb{R}^{2}}\langle f(t, x, v(x))-f(t, x, u(x)), z(x)\rangle \mathrm{d} x\right| \\
& \leq C_{1} a_{1}(t) \int_{\mathbb{R}^{2}}|v(x)-u(x) \| z(x)| \mathrm{d} x \\
& \leq C_{1} a_{1}(t)|v-u|_{L^{q}}|z|_{L^{q^{*}}},
\end{aligned}
$$

and consequently $F$ satisfies (ii). We will show that

$$
G(t, v)(\psi)(x)=\sum_{j=1}^{m} g^{j}(t, x, v(x)) \psi_{j}(x)
$$

has the required properties. Note that $G(t, v)$ is of the form (4.4) with

$$
u_{j}=g^{j}(t, \cdot, v(\cdot))=\tilde{G}_{j}(t, v) \text {. }
$$

Let

$$
\tilde{G}(t, v)(x)=\left(\tilde{G}_{1}(t, v)(x), \ldots, \tilde{G}_{m}(t, v)(x)\right) .
$$


Then using the same calculations as for $F$ we obtain $\tilde{G}(t, v) \in W^{1, q}\left(\mathbb{R}^{2} ; \mathbb{R}^{m}\right)$ and

$$
\|\tilde{G}(t, v)\|_{W^{1, q}\left(\mathbb{R}^{2} ; \mathbb{R}^{m}\right)} \leq C a_{2}(t)\left(1+\|v\|_{W^{1, q}}\right),
$$

with a certain $a_{2} \in L^{r}(0, T)$. Thus by Lemma 7.1, $G$ satisfies (i). We will show below that $G(t, \cdot)$ is a Lipschitz mapping from $L^{2}$ into $L_{(\mathrm{HS})}\left(\mathscr{H}_{\mathscr{W}}, L^{2}\right)$. This obviously guarantees that $G$ satisfies the condition (ii) of Theorem 2.1. Let $u, v \in L^{2}$ and $t \in[0, T]$. For $j=1, \ldots, m$ let $\left\{f_{k}^{j}\right\}$ be an orthonormal basis of $L_{(\mathrm{s})}^{2}\left(\mu_{j}\right)$. Then

$$
\begin{aligned}
& \|G(t, v)-G(t, u)\|_{L_{(\mathrm{HS})}\left(\not g_{y}, L^{2}\right)}^{2} \\
& \quad=\sum_{j, k} \int_{\mathbb{R}^{2}} \mid\left(g^{j}(t, x, v(x))-\left.g^{j}(t, x, u(x)) \widehat{f_{k}^{j} \mu}(x)\right|^{2} \mathrm{~d} x\right. \\
& \quad=\sum_{j, k} \frac{1}{4 \pi^{2}} \int_{\mathbb{R}^{2}} \mid \int_{\mathbb{R}^{2}} \mathrm{e}^{i\langle x, y\rangle}\left(g^{j}(t, x, v(x))-\left.g^{j}(t, x, u(x)) f_{k}^{j}(y) \mathrm{d} \mu_{j}(y)\right|^{2} \mathrm{~d} x\right. \\
& \quad=\sum_{j} \frac{1}{4 \pi^{2}} \int_{\mathbb{R}^{2}} \int_{\mathbb{R}^{2}} \mid \mathrm{e}^{i\langle x, y\rangle}\left(g^{j}(t, x, v(x))-\left.g^{j}(t, x, u(x))\right|^{2} \mathrm{~d} \mu_{j}(y) \mathrm{d} x\right. \\
& =\frac{1}{4 \pi^{2}} \sum_{j} \mu_{j}\left(\mathbb{R}^{2}\right) \int_{\mathbb{R}^{2}} \mid g^{j}(t, x, v(x))-g^{j}\left(t, x,\left.u(x)\right|^{2} \mathrm{~d} x\right. \\
& \quad \leq C a_{2}^{2}(t)|u-v|_{L^{2}}^{2},
\end{aligned}
$$

and the proof is complete.

8. Proof of Theorem 2.3. In the lemma below $W$ is an $L^{2}\left(\mathscr{O} ; \mathbb{R}^{m}\right)$-valued Wiener process with the covariance operator $Q$. By $\mathscr{Q}$ we denote the integral kernel corresponding to $Q^{1 / 2}$; see (2.3).

LEMMA 8.1. Let $r \in[0, \infty)$, and let $u \in W^{r, p}\left(\mathscr{O} ; \mathbb{R}^{m}\right)$. Let $\mathscr{H}$ be the RKHS of $W$, and let $K(u)$ be given by (4.4). Assume that

$$
\kappa(u, q, Q):=\sum_{l, j=1}^{m} \sum_{|\alpha+\beta| \leq 1} \int_{\mathscr{O}}\left|\partial^{\alpha} u_{j}(x)\right|^{q}\left(\int_{\mathscr{C}}\left(\partial_{x}^{\beta} \mathscr{Q}_{l, j}(x, y)\right)^{2} \mathrm{~d} y\right)^{q / 2} \mathrm{~d} x<\infty,
$$

Then $K(u) \in R\left(\mathscr{H}, W^{1, q}\right)$. Moreover, there is an independent of $u$ constant $C$ such that

$$
\|K(u)\|_{R\left(\mathscr{C}, W^{1, q}\right)} \leq C \kappa(u, q, Q)^{1 / q} .
$$

Proof. Recall (see Example 4.2) that $Q^{1 / 2}$ is an isomorphism between $\left(\operatorname{Ker} Q^{1 / 2}\right)^{\perp}$ and $\mathscr{H}$. Thus $K(u)$ is radonifying iff $K(u) Q^{1 / 2}$ is radonifying. Moreover,

$$
\|K(u)\|_{R\left(\mathscr{H} ; W^{1, q}\right)}=\left\|K(u) Q^{1 / 2}\right\|_{R\left(L^{2}\left(\mathscr{O} ; \mathbb{R}^{m}\right), W^{1, q}\right)} .
$$


Now note that

$$
\left(K(u) Q^{1 / 2} \psi\right)(x)=\sum_{j=1}^{m} u_{j}(x) \sum_{l=1}^{m} \int_{\mathscr{O}} \mathscr{Q}_{j, l}(x, y) \psi_{l}(y) \mathrm{d} y=\langle\mathscr{K}(u)(x), \psi\rangle_{L^{2}\left(\mathscr{O} ; \mathbb{R}^{m}\right)},
$$

where

$$
\mathscr{K}(u)(x)=\left(\sum_{j=1}^{m} u_{j}(x) \mathscr{Q}_{j, 1}(x, \cdot), \ldots, \sum_{j=1}^{m} u_{j}(x) \mathscr{Q}_{j, d}(x, \cdot)\right) .
$$

Furthermore, we have

$$
\|\mathscr{K}(u)\|_{W^{1, q}\left(\mathscr{C} ; L^{2}\left(\mathscr{O} ; \mathbb{R}^{m}\right)\right)}^{q} \leq C_{1} \kappa(u, q, Q),
$$

where $C_{1}$ is independent of $u$. Hence Theorem 4.1 yields the desired conclusion.

Proof of Theorem 2.3. It follows from the proof of Theorem 2.2 that $F$ given by (2.2) satisfies the conditions of Theorem 2.1. The proof of the fact that $G$ and $G$ given by (2.6) satisfies conditions (i) and (ii) of Theorem 2.1 follows the lines of the corresponding part of the proof of Theorem 2.2. The only difference being that instead of applying Lemma 7.1 now we use Lemma 8.1.

\section{APPENDIX A}

We shall deduce Theorem 3.1 from the following result.

Theorem A.1. There exists $\varepsilon<\frac{\pi}{2}$ and a constant $C_{\varepsilon}>0$ such that

$$
\left\|A_{q}^{i s}\right\|_{L\left(\mathbb{X}_{q}, \mathbb{X}_{q}\right)} \leq C_{\varepsilon} \mathrm{e}^{\varepsilon|s|}, \quad s \in \mathbb{R} .
$$

Proof. In the case $\mathscr{O}=\mathbb{R}^{2}$ the result is known (see, e.g., Lemma 5.3 in [26]).

If $\mathscr{O}$ is a proper subset of $\mathbb{R}^{2}$ we shall prove Theorem A. 1 by reducing it to an analogous result for the Laplace operator with Dirichlet boundary conditions in the $L^{q}$-space. Assumption A will play a crucial role. Namely let $B_{q}$ be the linear operator in the space $W^{-1, q}$ defined by

$$
\left\{\begin{array}{l}
B_{q} v=-\Delta v, v \in \operatorname{Dom}\left(B_{q}\right), \\
\operatorname{Dom}\left(B_{q}\right)=\left\{v \in W^{1, q}: v=0 \text { on } \partial D\right\} .
\end{array}\right.
$$

It follows from Assumption A that $\hat{B}_{q}^{-1}$ extends to a bounded and linear operator (in fact to an isomorphism) from $W^{-1, q}$ to $W_{0}^{1, q}$. Moreover, the inverse 
of such extension is exactly the operator $B_{q}$. Since also $\hat{B}_{q}^{-1 / 2}$ extends to an isomorphism (denoted by $J_{q}$ ) between $L^{q}$ and $W^{-1, q}$, the identity

$$
\left(\lambda+B_{q}\right)^{-1}=J_{q}^{-1}\left(\lambda+\hat{B}_{q}\right)^{-1} J_{q}, \quad \lambda \in \rho\left(B_{q}\right)
$$

proves that $B_{q}$ satisfies the statement of Theorem A.1, that is, there are $\varepsilon>0$ and $C_{\varepsilon}<\frac{\pi}{2}$ such that

$$
\left\|B_{q}^{i s}\right\|_{L\left(W^{-1, q}, W^{-1, q}\right)} \leq C_{\varepsilon} \mathrm{e}^{\varepsilon|s|}, \quad s \in \mathbb{R} .
$$

Suppose that $\lambda \in \mathbb{C}, f \in \mathbb{X}_{q}$ and $u \in \operatorname{Dom}\left(A_{q}\right)$ are such that $\left(\lambda+A_{q}\right) u=f$. Thus, there exists a function $p: \mathscr{O} \rightarrow \mathbb{R}$ such that

$$
(\lambda-\Delta) u+\nabla p=f \text { in } \mathscr{O}, \quad \operatorname{div} u=0 \text { in } \mathscr{O}, \quad\langle u, \mathbf{n}\rangle=0=\operatorname{curl} u \text { on } \partial \mathscr{O} .
$$

Then the function $v=\operatorname{curl} u$ is a solution to the following inhomogeneous Dirichlet boundary value problem

$$
(\lambda-\Delta) v=\operatorname{curl} f \text { in } \mathscr{O} \quad \text { and } \quad v=0 \text { on } \partial \mathscr{O} .
$$

The above argument can be reversed. Suppose that $f \in \mathbb{X}_{q}$ and that $v$ is a solution to (A.3). We need to find $u \in \operatorname{Dom}\left(A_{q}\right)$ such that curl $u=v$. For this let $z$ be the unique solution to

$$
-\Delta z=v \text { in } \mathscr{O} \quad \text { and } \quad z=0 \text { on } \partial \mathscr{O} .
$$

Let $u=\left(\partial_{2} z,-\partial_{1} z\right)$. Then:
(i) $u \in W^{2, q}$;
(ii) $\operatorname{div} u=0$;
(iii) $\langle u, \mathbf{n}\rangle=0$ on $\partial \mathscr{O}$;
(iv) $\operatorname{curl} u=0$ on $\partial \mathscr{O}$.

To prove the first property let us observe that since curl $f \in W^{-1, q}$, it follows from the elliptic regularity that $v \in W^{1, q}$. Hence, $z \in W^{3, q}$ and therefore $u \in$ $W^{2, q}$. The second follows as $\operatorname{div} u=\operatorname{div}\left(\partial_{2} \phi,-\partial_{1} \phi\right)=0$. The prove the third we first notice that $\langle u, \mathbf{n}\rangle=0$ on $\partial \mathscr{O}$, as $z=0$ on $\partial \mathscr{O}$. Therefore the vector field $\left(\partial_{2} \phi,-\partial_{1} \phi\right)$ is tangent to the boundary $\partial \mathscr{O}$ and hence (iii) follows. We finish by observing that in view of (A.3), (A.4), and the identity curl $\left(\partial_{2},-\partial_{1}\right)=-\Delta$, we have curl $u=-\Delta \phi=v=0$ on $\partial \mathscr{O}$. The properties (i) - (iv) proven above imply that the function $u$ satisfies the three boundary conditions from (A.2). We will find a function $p \in W^{1, q}$ such that the pair $(u, p)$ is a solution to (A.2). For this let $p \in W^{1, q}$ be a solution of

$$
\Delta p=\operatorname{div} \Delta u \text { in } \mathscr{O} \quad \text { and } \quad \frac{\partial p}{\partial \mathbf{n}}=0 \text { on } \partial \mathscr{O} .
$$

Then, arguing as in [45], we infer that the $(u, p)$ is a solution to (A.2).

Summing up we have shown that

$$
\left(\lambda+A_{q}\right)^{-1} f=\left(\partial_{2},-\partial_{1}\right) B_{q}^{-1}\left(\lambda+B_{q}\right)^{-1} \operatorname{curl} f
$$

for all $f \in \mathbb{X}_{q}$ and $\lambda \in \mathbb{C}$ from the resolvent set of $-B_{q}$. Since in view of Assumption $\mathrm{A},\left(\partial_{2},-\partial_{1}\right) B_{q}^{-1}$ is a bounded linear operator from $W^{-1, q}$ into $\mathbb{X}_{q}$ and 
curl is a bounded linear operator from $\mathbb{X}_{q}$ into $W^{-1, q}$, Theorem A.1 virtually follows from (A.1), (A.5) and the definition of imaginary powers.

Proof of Theorem 3.1(i). This result follows directly from Theorem A.1 and [39], Theorem 2.

Proof of Theorem 3.1(ii). This result follows directly from Theorem A.1 and [46], Theorem 1.15.3.

Proof of Theorem 3.1(iii). We know from Lemma 3.2 that $P_{q}$ is a bounded liner projection from $W^{q}$ onto $\mathbb{X}_{q}$. If $u \in W^{2, q}$ then $\operatorname{div} u \in W^{1, q}$ and hence by the elliptic regularity of Agmon-Douglis-Nirenberg (see [2] and [46]), the solution $p$ to (3.1) belongs to $W^{3, q}$. Hence $\mathscr{P}_{q} u \in W^{2, q}$. This argument shows also that the linear map $\mathscr{P}_{q}: W^{2, q} \rightarrow W^{2, q}$ is bounded. Hence the map $\mathscr{P}_{q}: W^{2, q} \rightarrow W^{2, q} \cap \mathbb{X}_{q}=H^{2, q}$ is bounded. Then by the RieszThorin theorem the map $\mathscr{P}_{q}:\left[W^{2, q}, L^{q}\right]_{r / 2} \rightarrow\left[H^{2, q}, \mathbb{X}_{q}\right]_{r / 2}$ is bounded as well. Therefore we infer the result by using equalities: $\left[W^{2, q}, L^{q}\right]_{r / 2}=W^{r, q}$ and $\left[H^{2, q}, \mathbb{X}_{q}\right]_{r / 2}=\left[\operatorname{Dom}\left(A_{q}\right), \mathbb{X}_{q}\right]_{r / 2}=\operatorname{Dom}\left(A_{q}^{r}\right)=H^{2 r, q}$. Note that the equality $\left[\operatorname{Dom}\left(A_{q}\right), \mathbb{X}_{q}\right]_{r / 2}=\operatorname{Dom}\left(A_{q}^{r}\right)$ follows from Theorem A.1 and [46], Theorem 1.15.3.

Proof of TheOREM 3.1(iv). Integrating by parts we get

$$
\left\langle A_{q} v, u\right\rangle=\int_{\mathscr{O}} \operatorname{curl} v(x) \operatorname{curl} u(x) \mathrm{d} x,
$$

and the desired conclusion follows from Hölder's inequality.

Proof of Theorem 3.2(i). Since $\mathscr{P}$ is continuous on $L^{q}$, we have

$$
|B(u, v)|_{q} \leq\left(|u|_{\infty}\|v\|_{W^{1, q}}+\|u\|_{W^{1, q}}|v|_{\infty}\right),
$$

and the desired conclusion follows from the continuity of the imbeddings $H^{r, q} \hookrightarrow W^{r, q}$, see Theorem 3.1(iii), and

$$
W^{\delta+1, q} \hookrightarrow L^{\infty}, \quad \delta>0
$$

(see, e.g., [44], Theorem 3.1, or [43], Theorem 2, page 124).

Proof of Theorem 3.2(ii). Let $q^{*}$ be the conjugate exponent to $q: \frac{1}{q}+\frac{1}{q^{*}}=$ 1. Clearly we may assume that $r<\frac{2}{q^{*}}$. Then $\frac{1}{q^{*}}-\frac{r}{2}>0$ and so we can choose $p>1$ such that $\frac{1}{p}=\frac{1}{q^{*}}-\frac{r}{2}$. Since then (as $\left.r>0\right) \frac{1}{q}+\frac{1}{p}=1-\frac{r}{2}<1$, we can find $s>1$ such that $\frac{1}{s}+\frac{1}{q}+\frac{1}{p}=1$. Let $u, v, z \in H^{1, q}$. In what follows the constants $c_{i}$ do not depend on $u, v, z$. First the Hölder inequality imply that

$$
|\langle B(u, v), z\rangle| \leq c_{1}|u|_{s}|\nabla v|_{q}|z|_{p} .
$$


Next, the above choice of $p$ and $s$ implies that the imbeddings $W^{1, q} \hookrightarrow L^{s}$ and $W^{r, q^{*}} \hookrightarrow L^{p}$ are continuous (see, e.g., [1], Lemma 5.14, or [43], Theorem 2, page 124), and therefore

$$
|\langle B(u, v), z\rangle| \leq c_{2}\|u\|_{W^{1, q}}\|v\|_{W^{1, q}}\|z\|_{W^{r, q^{*}}} \leq c_{3}\|u\|_{H^{1, q}}\|v\|_{H^{1, q}}\|z\|_{H^{r, q^{*}}} .
$$

Therefore, as $H^{-r, q}=\left(H^{r, q^{*}}\right)^{*}$, the proof is complete.

Proof of THEOREM 3.2(iii). This proof follows the lines of the proof of Theorem 3.2(i).

Proof OF Theorem 3.2(iv). The case $q=2$ is well known, see [45] and [3]. Assume hence that $q>2$. Since $H^{1,2} \cap H^{1, q}$ is dense in $H^{1, q}$ and by the Sobolev imbedding $H^{1, q} \hookrightarrow L^{q^{*}}$, we only need to show the desired equality for $u, v \in H^{1,2} \cap H^{1, q}$. But this follows from the case $q=2$.

Proof of Theorem 3.2(v). It is enough to show the identity for $u, v \in$ $C_{\text {sol }}^{\infty} \operatorname{such}$ that $\operatorname{curl} u=0$ and $\operatorname{curl} v=0$ on $\partial \mathscr{O}$. For such $u$ and $v$ we have $\operatorname{curl} B(u, v)=\operatorname{curl}[(u \cdot \nabla) v]=(u \cdot \nabla) \operatorname{curl} v$. Then, denoting $\varphi=\operatorname{curl} v$, we have by integration by parts

$$
\begin{aligned}
\left\langle(u \cdot \nabla) \varphi, \varphi|\varphi|^{q-2}\right\rangle & =-\left\langle(u \cdot \nabla)\left[\varphi|\varphi|^{q-2}\right], \varphi\right\rangle \\
& =-(q-1)\left\langle[(u \cdot \nabla) \varphi]|\varphi|^{q-2}, \varphi\right\rangle \\
& =-(q-1)\left\langle(u \cdot \nabla) \varphi, \varphi|\varphi|^{q-2}\right\rangle .
\end{aligned}
$$

Hence we obtain the desired conclusion.

\section{APPENDIX B}

The following result gathers the main properties of the space of radonifying operators. For the proof of the theorem below we refer the reader to Baxendale [4].

Theorem B.1. Let $\mathscr{H}$ and $E$ be real separable Hilbert and Banach spaces, respectively. Then for arbitrary $K \in R(\mathscr{H}, E)$ and orthonormal basis $\left\{e_{k}\right\}$ of $\mathscr{H}$ one has

$$
\left\|K-K \Pi_{n}\right\|_{R(\mathscr{H}, E)} \rightarrow 0 \quad \text { as } n \rightarrow \infty,
$$

where $\Pi_{n}$ stands for the projection of $\mathscr{H}$ onto the space spanned by $e_{1}, \ldots, e_{n}$.

Proof of ThEOREM 4.1. The first step is to show that there is a constant $C$ depending only on $r$ and $q$ such that for any $n \in \mathbb{N}$ and for any operator $K: \mathbb{R}^{n} \rightarrow W^{r, q}$ given by the kernel $\mathscr{K} \in W^{r, q}\left(\mathscr{O} ; \mathbb{R}^{n}\right)$ one has

$$
\|K\|_{R\left(\mathbb{R}^{n}, W^{r, q}\right)} \leq C\|\mathscr{K}\|_{W^{r, q}\left(\mathscr{O} ; \mathbb{R}^{n}\right)} .
$$


Let $\left\{e_{k}\right\}$ be an orthonormal basis of $\mathbb{R}^{n}$, and let $\left\{\beta_{k}\right\}_{k \in \mathbb{N}}$ be a system of independent identically distributed normal variables. We have to estimate

$$
\|K\|_{R\left(\mathbb{R}^{n}, W^{r, q}\right)}=\left(\mathbb{E}\left\|\sum_{k=1}^{n} \beta_{k} K e_{k}\right\|_{W^{r, q}}^{2}\right)^{1 / 2} .
$$

To do this we first assume that $r \in \mathbb{N} \cup\{0\}$. Then

$$
\begin{aligned}
\left(\mathbb{E}\left\|\sum_{k=1}^{n} \beta_{k} K e_{k}\right\|_{W^{r, q}}^{2}\right)^{q / 2} & \leq \mathbb{E}\left\|\sum_{k=1}^{n} \beta_{k} K e_{k}\right\|_{W^{r, q}}^{q} \\
& \leq \mathbb{E} \int_{\mathscr{O}} \sum_{|\alpha| \leq r}\left|\sum_{k=1}^{n} \beta_{k} \partial^{\alpha}\left(K e_{k}\right)(x)\right|^{q} \mathrm{~d} x .
\end{aligned}
$$

Using the normality of $\sum_{k} \beta_{k} \partial^{\alpha}\left(K e_{k}\right)(x)$, and the fact that there is a constant $C$ such that for an arbitrary centered normal random variable $\xi, \mathbb{E}|\xi|^{q} \leq$ $C\left(\mathbb{E}|\xi|^{2}\right)^{q / 2}$, we get

$$
\begin{aligned}
\|K\|_{R\left(\mathbb{R}^{n}, W^{r, q}\right)}^{q} & \leq C \int_{\mathscr{O}} \sum_{|\alpha| \leq r}\left(\mathbb{E}\left|\sum_{k=1}^{n} \beta_{k} \partial^{\alpha}\left(K e_{k}\right)(x)\right|^{2}\right)^{q / 2} \mathrm{~d} x \\
& \leq C \int_{\mathscr{O}} \sum_{|\alpha| \leq r}\left(\sum_{k=1}^{n}\left|\partial^{\alpha}\left(K e_{k}\right)(x)\right|^{2}\right)^{q / 2} \mathrm{~d} x \\
& \leq C \int_{\mathscr{O}} \sum_{|\alpha| \leq r}\left|\partial^{\alpha} \mathscr{K}(x)\right|_{\mathbb{R}^{n}}^{q} \mathrm{~d} x \leq C\|\mathscr{K}\|_{W^{r, q}\left(\mathcal{O} ; \mathbb{R}^{n}\right)}^{q} .
\end{aligned}
$$

Thus we have (B.1) for $l \in \mathbb{N} \cup\{0\}$. Using standard interpolation arguments one can show (B.1) for an arbitrary $r \in[0, \infty)$. Now let $\mathscr{H}$ be an infinite dimensional Hilbert space, let $\left\{e_{k}\right\}$ be an orthonormal basis of $\mathscr{H}$, and let $\Pi_{n}$ be the projection of $\mathscr{H}$ onto the space spanned by $e_{1}, \ldots, e_{n}$, which we identify with $\mathbb{R}^{n}$. Then, by Theorem B.1 we have

$$
\begin{aligned}
\|K\|_{R\left(\mathscr{H}, W^{r, q}\right)} & \leq \lim _{n \rightarrow \infty}\left\|K \Pi_{n}\right\|_{R\left(\mathscr{H}, W^{r, q}\right)} \leq C \lim _{n \rightarrow \infty}\left\|\Pi_{n} \mathscr{K}\right\|_{W^{r, q}\left(\mathscr{O} ; \mathbb{R}^{n}\right)} \\
& \leq C\|\mathscr{K}\|_{W^{r, q}(\mathscr{C} ; \mathscr{C})},
\end{aligned}
$$

which is the desired conclusion.

Acknowledgments. The authors would like to thank the referees for their valuable comments and pointing out numerous mistakes in the first version of the manuscript.

\section{REFERENCES}

[1] Adams, R. A. (1975). Sobolev Spaces. Academic Press, New York.

[2] Agmon, S., Douglis, A. and NiREnBerg, L. (1959). Estimates near boundary for solutions of elliptic partial differential equations satisfying general boundary conditions I. Comm. Pure Appl. Math. 12 623-727.

[3] BARDos, C. (1972). Existence et unicité de la solution de l'equation d'Euler en dimension deux. J. Math. Anal. Appl. 40 769-790. 
[4] BaXendale, P. (1976). Gaussian measures on function spaces. Amer. J. Math. 98 891-952.

[5] BerGH, J. and LöfströM, J. (1976). Interpolation Spaces. Springer, Berlin.

[6] BessaiH, H. (1999). Stochastic PDEs of Euler type. Ph.D. thesis, Scuola Normale Superiore, Pisa.

[7] Bessaih, H. and Flandoli, F. (1999). 2-D Euler equations perturbed by noise. Nonlinear Differential Equations and Applications 6 35-54.

[8] Bessait, H. (1999). Martingale solutions for stochastic Euler equations. Stochastic Anal. Appl. 17 713-725.

[9] BRZEŹNIAK, Z. (1995). Stochastic partial differential equations in $M$-type 2 Banach spaces. Potential Anal. 4 1-45.

[10] BRZEŹNIAK, Z. (1997). On stochastic convolutions in Banach spaces and applications. Stochastics Stochastics Rep. 61 245-295.

[11] BRZEŹNIAK, Z. and CARROLL, A. (2000). Approximations of the Wong-Zakai type for stochastic differential equations in $M$-type 2 Banach spaces with applications to loop spaces. Preprint.

[12] BRZEŹNiAK, Z. and GĄTAREK, D. (1999). Martingale solutions and invariant measures for stochastic evolution equations in Banach spaces. Stochastic Processes Appl. 87 187226.

[13] BrZeźniaK, Z. and PesZat, S. (1999). Space-time continuous solutions to SPDEs driven by a homogeneous Wiener process. Studia Math. 137 261-299.

[14] BrzeźniaK, Z. and Peszat, S. (2000). Strong local and global solutions to stochastic NavierStokes equations. In Infinite-Dimensional Stochastic Analysis 85-98. Royal Netherlands Academy of Arts and Sciences, Amsterdam.

[15] CAPiński, M. and Cutland, N. J. (1999). Stochastic Euler equations on the torus. Ann. Appl. Probab. 9 688-705.

[16] CAPIŃSKI, M. and GąTAREK, D. (1994). Stochastic equations in Hilbert space with applications to 3-D stochastic Navier-Stokes equations. J. Funct. Anal. 126 25-35.

[17] CAPiŃski, M. and PEsZAT, S. (2001). On the existence of a solution to stochastic NavierStokes equations. Nonlinear Anal. 44 141-177.

[18] Carroll, A. (1999). The stochastic nonlinear heat equation. Ph.D. thesis, Univ. Hull.

[19] DA PRATO, G. and ZABCZYK. J (1992). Stochastic Equations in Infinite Dimensions. Cambridge Univ. Press.

[20] Da Prato, G. and ZabczyK, J. (1996). Ergodicity for Infinite Dimensional Systems. Cambridge Univ. Press.

[21] Dawson, D. A. and SAlEHI, H. (1980). Spatially homogeneous random evolutions. J. Multivariate Anal. 10 141-180.

[22] DetTweiler, E. (1991). Stochastic integration relative to Brownian motion on a general Banach space. Doğa Mat. 15 6-44.

[23] Fujiwara, D. and Morimoto, H. (1977). An $L_{r}$ theorem of the Helmhotz decomposition of vector fields. J. Fac. Sci. Univ. Tokyo Sect. IA Math. 24 685-700.

[24] GątareK, D. and GoŁdys, B. (1993). On weak solutions of stochastic equations in Hilbert spaces. Stochastics Stochastic Rep. 46 41-51.

[25] Giga, Y. (1985). Domains of fractional powers of the Stokes operator in $L^{r}$ spaces. Arch. Ration. Mech. Anal. 89 251-265.

[26] GigA, Y. and SoHR, H. (1989). On the Stokes operator in exterior domain. J. Fac. Sci. Univ. Tokyo Sect. IA Math. 36 103-130.

[27] Ikeda, N. and Watanabe, S. (1981). Stochastic Differential Equations and Diffusion Processes. North-Holland, Amsterdam.

[28] Iтô, K. (1984). Foundations of Stochastic Differential Equations in Infinite Dimensional Spaces. SIAM, Philadelphia.

[29] Kato, T. and Ponce, G. (1986). Well-posedness of the Euler and Navier-Stokes equations in Lebesgue spaces $L_{s}^{p}\left(\mathbb{R}^{n}\right)$. Rev. Mat. Iberoamericana 2 73-88.

[30] KATo, T. and Ponce, G. (1987). On nonstationary flows of viscous and ideal fluid in $L_{s}^{p}\left(\mathbb{R}^{2}\right)$. Duke Math. J. 55 487-499. 
[31] Kuo, H. H. (1975). Gaussian Measures in Banach Spaces. Lecture Notes in Math. 463. Springer, Berlin.

[32] Kwapień, S. and Woyczynski, W. A. (1992). Random Series and Stochastic Integrals: Single and Multiple. Birkhäuser, Boston.

[33] Lions, P. L. (1996). Mathematical Topics in Fluid Mechanics 1. Oxford Science Publishers.

[34] Metivier, M. (1988). Stochastic Partial Differential Equations in Infinite Dimensional Spaces. Scuola Normale Superiore, Pisa.

[35] MÉtivieR, M. and NAKAO, S. (1987). Equivalent conditions for the tightness of a sequence of continuous Hilbert valued martingales. Nagoya J. Math. 106 113-119.

[36] NeidHARDT, A. L. (1978). Stochastic integrals in 2-uniformly smooth Banach spaces. Ph.D. dissertation, Univ. Wisconsin.

[37] PARDoux, E. (1975). Equations aux derivées partielles stochastiques nonlinéaires monotones. Thèse, Univ. Paris XI.

[38] Peszat, S. and ZaBCZYK, J. (1997). Stochastic evolution equations with a spatially homogeneous Wiener process. Stochastic Processes Appl. 72 187-204.

[39] PRÜsS, J and SoHR, H. (1990). On operators with bounded imaginary powers. Math. Z. 203 $429-452$.

[40] PRÜss, J. and SoHR, H. (1993). Imaginary powers of elliptic second order differential operators in $L^{p}$-spaces. Hiroshima Math. J. 23 161-192.

[41] RozovskiI, B. L. (1990). Stochastic Evolution Equations. Linear Theory and Applications to Non-linear Filtering. Kluwer, Dordrecht.

[42] Seeley, R. (1971). Norms and domains of complex powers $A_{B}^{z}$. Am. J. Math. 93 299-309.

[43] Stein, E. (1970). Singular Integrals and Differentiability Properties of Functions. Princeton Univ. Press.

[44] TAYlor, M. E. (1981). Pseudodifferential Operators. Princeton Univ. Press.

[45] Temam, R. (1979). Navier-Stokes Equations, Theory and Numerical Analysis. North-Holland, Amsterdam.

[46] TRIEBel, H. (1978). Interpolation Theory, Function Spaces, Differential Operators. NorthHolland, Amsterdam.

[47] Vishik, M. I. and Fursikov, A. V. (1988). Mathematical Problems of Statistical Hydromechanics. Kluwer, Dordrecht.

DEPARTMENT OF MATHEMATICS

UNIVERSITY OF HULL

HULL HU6 7RX

ENGLAND

E-MAIL: Z.Brzezniak@maths.hull.ac.uk
INSTITUTE OF MATHEMATICS

Polish ACADEMY OF SCIENCES

Św. TOMASZA 30/7

31-027 KRAKÓW

POLAND

E-MAIL: napeszat@cyf-kr.edu.pl 